\title{
Article \\ Ethanol Treatment Enhances Physiological and Biochemical Responses to Mitigate Saline Toxicity in Soybean
}

\author{
Ashim Kumar Das ${ }^{1,+}$ (D), Touhidur Rahman Anik ${ }^{2,+}$, Md. Mezanur Rahman ${ }^{3,+} \mathbb{D}$, Sanjida Sultana Keya ${ }^{3}$, \\ Md. Robyul Islam ${ }^{4}$ (D, Md. Abiar Rahman ${ }^{1}$, Sharmin Sultana ${ }^{4}$, Protik Kumar Ghosh ${ }^{5}$, Sabia Khan ${ }^{6}$, \\ Tofayel Ahamed ${ }^{1}$, Totan Kumar Ghosh ${ }^{7}$ (D), Lam Son-Phan Tran ${ }^{3}$ (D) and Mohammad Golam Mostofa ${ }^{3,8, *}$ (ID
}

Citation: Das, A.K.; Anik, T.R.; Rahman, M.M.; Keya, S.S.; Islam, M.R.; Rahman, M.A.; Sultana, S.; Ghosh, P.K.; Khan, S.; Ahamed, T.; et al. Ethanol Treatment Enhances Physiological and Biochemical Responses to Mitigate Saline Toxicity in Soybean. Plants 2022, 11, 272. https://doi.org/10.3390/plants 11030272

Academic Editor: Raffaella Maria Balestrini

Received: 21 November 2021 Accepted: 11 January 2022 Published: 20 January 2022 Publisher's Note: MDPI stays neutral with regard to jurisdictional claims in published maps and institutional affiliations.

Copyright: (C) 2022 by the authors. Licensee MDPI, Basel, Switzerland. This article is an open access article distributed under the terms and conditions of the Creative Commons Attribution (CC BY) license (https:// creativecommons.org/licenses/by/ $4.0 /)$.
1 Department of Agroforestry and Environment, Bangabandhu Sheikh Mujibur Rahman Agricultural University, Gazipur 1706, Bangladesh; ashimbsmrau@gmail.com (A.K.D.); abiar@bsmrau.edu.bd (M.A.R.); tofayela@gmail.com (T.A.)

2 Plant Pathology Division, Bangladesh Rice Research Institute, Gazipur 1701, Bangladesh; anikbge@gmail.com

3 Department of Plant and Soil Science, Institute of Genomics for Crop Abiotic Stress Tolerance, Texas Tech University, Lubbock, TX 79409, USA; mdmerahm@ttu.edu (M.M.R.); skeya@ttu.edu (S.S.K.); son.tran@ttu.edu (L.S.-P.T.)

4 Institute of Biotechnology and Genetic Engineering (IBGE), Bangabandhu Sheikh Mujibur Rahman Agricultural University, Gazipur 1706, Bangladesh; irobyul@gmail.com (M.R.I.); sharminomi87@gmail.com (S.S.)

5 Faculty of Agriculture, Bangabandhu Sheikh Mujibur Rahman Agricultural University, Gazipur 1706, Bangladesh; protikbsmrau@gmail.com

6 Department of Plant Pathology, Bangabandhu Sheikh Mujibur Rahman Agricultural University, Gazipur 1706, Bangladesh; sabia.khan60@gmail.com

7 Department of Crop Botany, Bangabandhu Sheikh Mujibur Rahman Agricultural University, Gazipur 1706, Bangladesh; totan@bsmrau.edu.bd

8 Department of Biochemistry and Molecular Biology, Bangabandhu Sheikh Mujibur Rahman Agricultural University, Gazipur 1706, Bangladesh

* Correspondence: mostofa@bsmrau.edu.bd or mmostofa@ttu.ed

+ These authors contributed equally to this work.

\begin{abstract}
Soil salinity, a major environmental concern, significantly reduces plant growth and production all around the world. Finding solutions to reduce the salinity impacts on plants is critical for global food security. In recent years, the priming of plants with organic chemicals has shown to be a viable approach for the alleviation of salinity effects in plants. The current study examined the effects of exogenous ethanol in triggering salinity acclimatization responses in soybean by investigating growth responses, and numerous physiological and biochemical features. Foliar ethanol application to saline water-treated soybean plants resulted in an enhancement of biomass, leaf area, photosynthetic pigment contents, net photosynthetic rate, shoot relative water content, water use efficiency, and $\mathrm{K}^{+}$and $\mathrm{Mg}^{2+}$ contents, leading to improved growth performance under salinity. Salt stress significantly enhanced the contents of reactive oxygen species (ROS), malondialdehyde, and electrolyte leakage in the leaves, suggesting salt-induced oxidative stress and membrane damage in soybean plants. In contrast, ethanol treatment of salt-treated soybean plants boosted ROS-detoxification mechanisms by enhancing the activities of antioxidant enzymes, including peroxidase, ascorbate peroxidase, catalase, and glutathione $S$-transferase. Ethanol application also augmented the levels of proline and total free amino acids in salt-exposed plants, implying a role of ethanol in maintaining osmotic adjustment in response to salt stress. Notably, exogenous ethanol decreased $\mathrm{Na}^{+}$uptake while increasing $\mathrm{K}^{+}$and $\mathrm{Mg}^{2+}$ uptake and their partitioning to leaves and roots in salt-stressed plants. Overall, our findings reveal the protective roles of ethanol against salinity in soybean and suggest that the use of this cost-effective and easily accessible ethanol in salinity mitigation could be an effective approach to increase soybean production in salt-affected areas.
\end{abstract}

Keywords: antioxidants; ethanol; ionic balance; oxidative damage; photosynthesis; reactive oxygen species; salinity; soybean 


\section{Introduction}

Climate change-induced extreme environmental conditions, such as salinity, drought, temperature extremes, and waterlogging, pose serious challenges to global agriculture, threatening food security [1]. Furthermore, given the current water-scarce scenario, high saline water is increasingly being used to meet the growing need for water for agricultural production in many parts of the world [2,3]. Consequently, an increasing level of soil salinity has emerged as a paramount environmental problem, affecting around 3.6 billion hectares (Bha) out of 5.22 Bha of the world's agricultural land, with an annual loss of USD 27.5 billion $[2,4,5]$. Plants grown in salt-contaminated soils have accumulated excessive toxic ions, resulting in a variety of morphological, physiological, and biochemical disturbances, such as ionic disparities, poor gas exchange performance, photosynthetic pigments loss, low water status, and excessive reactive oxygen species (ROS)-induced oxidative damage to cellular components [2,6-9]. Plants, on the other hand, employ a wide array of mechanisms to counteract the detrimental consequences of salt stress. For example, salt-exposed plants mount mechanisms to (i) reduce toxic ion accumulations in the aerial parts; (ii) limit the destruction of photosynthetic pigments; (iii) increase the accumulation of osmoprotectants, such as proline (Pro), free amino acids and soluble sugars; and (iv) enhance the activities of antioxidant enzymes, including superoxide dismutase (SOD), ascorbate peroxidase (APX), catalase (CAT), glutathione S-transferase (GST), and glutathione peroxidase (GPX), and the contents of nonenzymatic compounds, such as carotenoids, phenolic compounds, and flavonoids $[2,7,8]$.

Soybean (Glycine max) is a nutritious, low-cost, and economically important crop belonging to the family Fabaceae, which contributes to nearly $70 \%$ of plant-based protein consumption and $29 \%$ of edible oil worldwide [10]. Furthermore, being a legume crop, the inclusion of soybean in crop rotation is an effective approach to replenishing soil fertility, strengthening soil-nutrient recycling, improving soil microbial activities, and increasing crop yield due to its biological nitrogen $(\mathrm{N})$ fixation ability [11]. Due to its numerous benefits, soybean has become a globally coveted crop, and its demand is steadily increasing year to year [10]. In Bangladesh, soybean is cultivated on roughly 0.07734 million ha (Mha) of land out of 0.47874 Mha of the total oil-cropped areas [12], and soybean production is estimated to be around 0.14695 million tons (MT) every year [13]. The growing population, changing consumer eating habits, expansion of the bakery and food industries, and increasing need for soy meal for the livestock and fishing sectors are among the major factors driving up demand for soybean products in Bangladesh [14]. Bangladesh's agricultural production systems were unable to produce enough soybeans, resulting in the import of 2.4 MT of soybeans in 2020, which is expected to rise to 2.65 MT in 2021-2022 (https:/ / www.fas.usda.gov/data/bangladesh-oilseeds-and-products-annual-2) (accessed on 10 November 2021). In addition to limited arable land for soybean cultivation, soil salinity caused a significant decline in soybean production and seed quality in Bangladesh [15]. Elevated soil salinity perturbs the entire life cycle of soybean, from germination to plant growth, and the formation of nodule and seed yield [10], although the seedling stage of soybean is more vulnerable to salinity than the germination stage [16]. It is reported that salinity can impede soybean seed germination by $18 \%$ (at $2.09 \mathrm{dS} \mathrm{m}^{-1}$ ) to $70 \%$ (at $>5 \mathrm{dS} \mathrm{m}^{-1}$ ) in salt-affected areas of Bangladesh [17].

To improve the salinity tolerance of soybean, various approaches, including gene discovery, breeding, and biotechnological strategies, are in practice [18]. Apart from the aforementioned strategies, developing simple and less expensive technologies for low-income nations such as Bangladesh, where insufficient funding in research and development discourages scientists from researching, and developing genetically modified crops, is still necessary. In this context, exploring the potential roles of external chemicals may offer an effective strategy in boosting plant resiliency against ever-changing environmental assaults. Ethanol has been demonstrated to improve chilling tolerance in rice (Oryza sativa) [19] and salt stress tolerance in rice and Arabidopsis thaliana [20]. The application of ethanol augmented the chlorophyll (Chl) content in Arabidopsis under salt stress while simultaneously 
lowering the buildup of ROS [20]. In line with this, exogenous ethanol supplementation has also been shown to upregulate the expressions of $A P X 1$ and APX2, encoding APX, and ROS signaling-related transcription factor genes ZAT10 and ZAT12, which were associated with salinity acclimatization responses in Arabidopsis [20]. Considering this clue, we also foresee that this affordable and easily accessible chemical ethanol could play an influential role in reducing salt-induced adverse effects on an economically important crop, soybean.

In this study, we examined whether ethanol could protect soybeans, as it did in other crops, from the adverse effects of salinity. If it does so, what are the underlying mechanisms that play vital roles in improving the salt tolerance potential of soybeans? Here, we examined the functions of ethanol in improving the salinity tolerance of soybean by evaluating the morphological, physiological, and biochemical features associated with (i) growth enhancement and biomass production, (ii) sodium ion $\left(\mathrm{Na}^{+}\right)$uptake and accumulation, (iii) photosynthetic pigment status, (iv) salt-induced oxidative stress, (v) antioxidant defense system, and (vi) osmotic adjustments.

\section{Results}

\subsection{Ethanol Improves Phenotypic Appearance of Soybean Plants}

Soybean plants exposed to $8 \mathrm{dS} \mathrm{m}^{-1}$ (S1) and $16 \mathrm{dS} \mathrm{m} \mathrm{m}^{-1}$ (S2) salt stress for 7 days showed substantial phenotypic disruption, including stunted growth, wilting, early senescence, decolorization of leaves (turned to pale and yellow), and reduction in root length, as compared with the 'Control' plants (Figure 1a-d). By contrast, plants sprayed with $20 \mathrm{mM}$ of ethanol (Eth) significantly reduced salinity-induced toxic effects in 'S1 + Eth' and 'S2 + Eth' plants, as manifested by their improved phenotypes, including less wilting and yellowing of leaves, delayed leaf senescence, and enhanced root length, when equated with the respective salt-stressed 'S1' and 'S2' plants (Figure 1a-d). Interestingly, under non-stressed conditions, the application of exogenous ethanol also improved the visual appearance of the shoots and roots in 'Eth' plants, in relation to the 'Control' plants (Figure 1a-d).
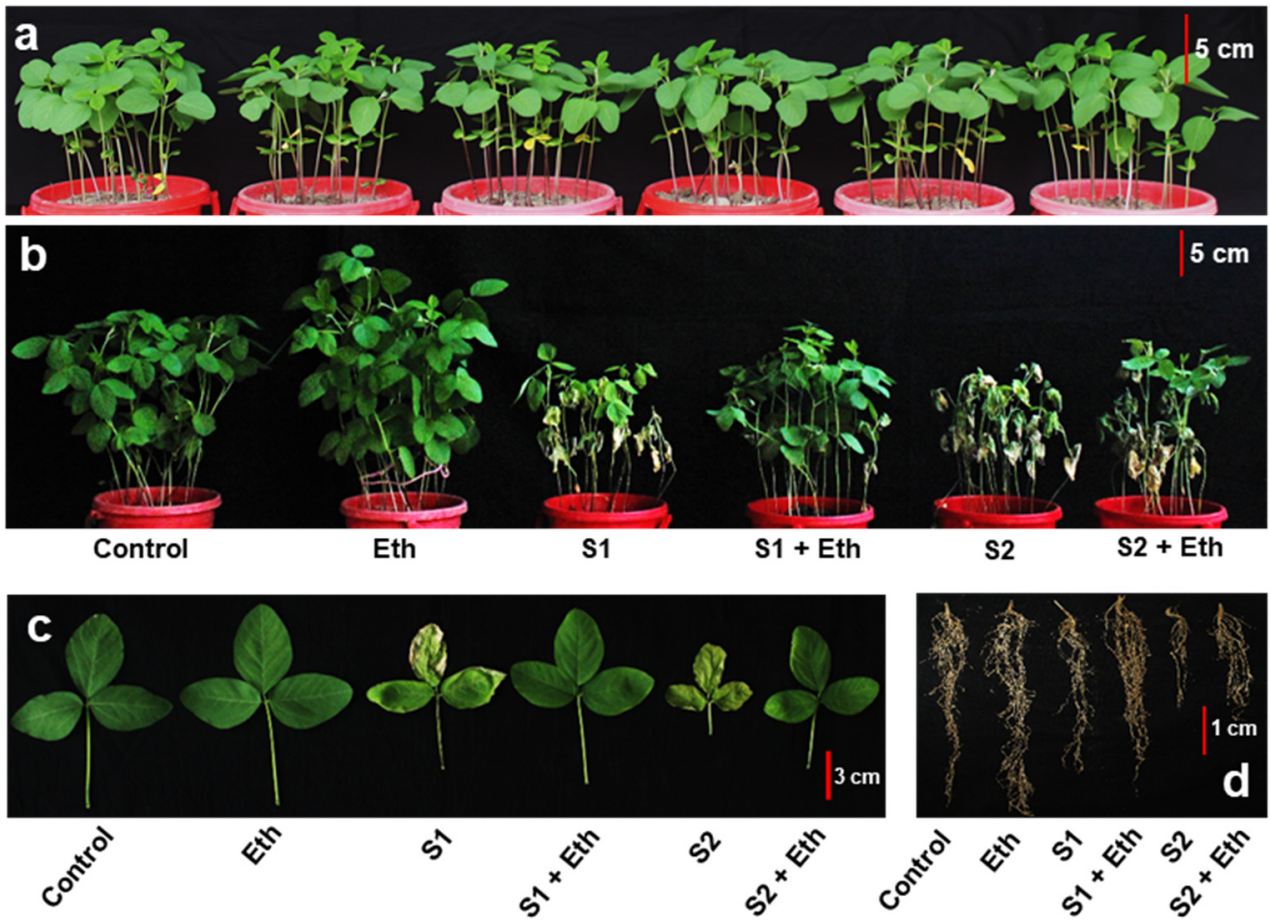

Figure 1. Cont. 

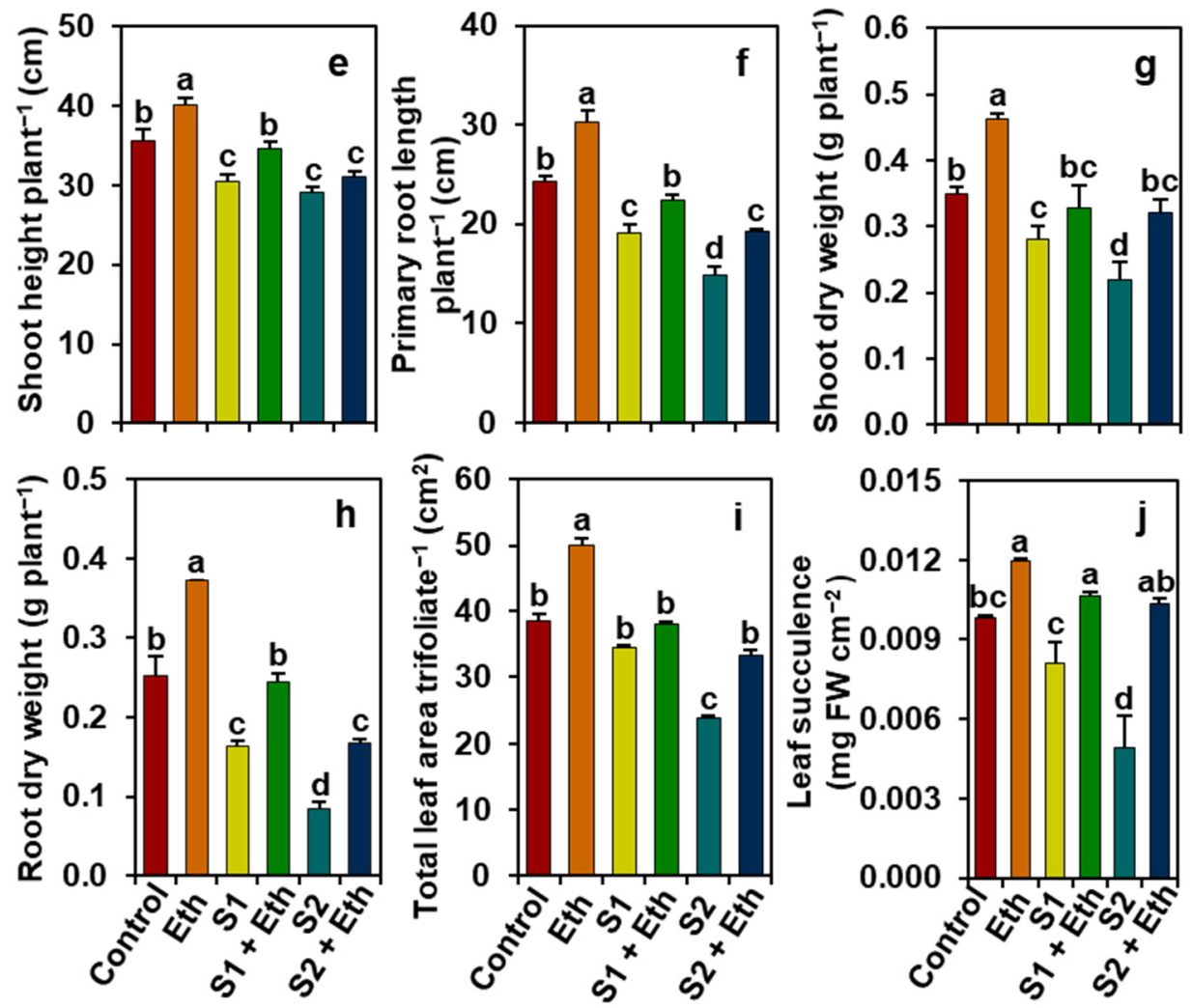

Figure 1. Effects of foliar-sprayed ethanol on growth-associated attributes of soybean plants under salt stress conditions. Representative photographs of soybean plants before stressed with salt (a) and after exposure to a gradient of salinity for 7 days (b). Close view of representative leaves (c) and roots (d) showing a positive effect of ethanol on salt-stressed soybean plants. (e) Shoot height, (f) primary root length, (g) shoot DW, (h) root DW, (i) total leaf area per trifoliate, and (j) leaf succulence of soybean plants exposed to different levels of salinity for 7 days in the presence and absence of ethanol. The statistically significant differences $(p<0.05)$ among various treatments are shown by different letters following the least significant difference (LSD) test. FW, fresh weight; DW, dry weight; Eth, $20 \mathrm{mM}$ ethanol; S1, $8 \mathrm{dS} \mathrm{m}^{-1}$; S1 + Eth, $8 \mathrm{dS} \mathrm{m}^{-1}+20 \mathrm{mM}$ ethanol; S2, $16 \mathrm{dS} \mathrm{m}^{-1}$; S2 + Eth, $16 \mathrm{dS} \mathrm{m}^{-1}+20 \mathrm{mM}$ ethanol.

\subsection{Ethanol Boosts Growth Attributes in Salt-Stressed Soybean Plants}

'S1' and 'S2' plants, respectively, displayed noteworthy reductions in shoot height (by 14.41 and $18.34 \%$ ), root length (21.37 and $39.04 \%)$, shoot dry weight (DW) (19.52 and $37.14 \%)$, root DW (35.10 and $66.23 \%)$, total leaf area per trifoliate $(38.19 \%$ in 'S2'), and leaf succulence $(50.10 \%$ in 'S2'), when compared with those of the 'Control' plants (Figure 1e-j respectively). Conversely, remarkable improvements in shoot height (by 13.66\% in 'S1 + Eth'), root length (16.72 and 29.89\%), shoot DW (45.45\% in 'S2 + Eth'), root DW (48.98 and $96.08 \%)$, total leaf area per trifoliate (39.75\% in 'S2 + Eth'), and leaf succulence (32.05 and 110.68\%) were manifested in 'S1 + Eth' and 'S2 + Eth' plants, respectively, when contrasted with the corresponding 'S1' and 'S2' plants (Figure 1e-j). Moreover, 'Eth' plants also showed enhancements in the height of shoot, length of root, root DW, shoot DW, total leaf area per trifoliate, and leaf succulence by $12.35,24.25,47.68,32.38,29.96$, and 21.76\%, respectively, compared with the corresponding data obtained from the 'Control' plants (Figure 1e-j).

\subsection{Ethanol Protects Gas Exchange Features in Soybean Plants under Salt Stress}

Relative to the 'Control' plants, 'S1' and 'S2' plants, respectively, showed remarkably decreased levels of photosynthetic rate $\left(P_{n}\right.$, by 74.03 and $\left.96.81 \%\right)$, stomatal conductance to $\mathrm{H}_{2} \mathrm{O}\left(g_{s}, 91.12\right.$ and $\left.97.93 \%\right)$, transpiration rate $(E, 66.57$ and $85.66 \%)$, and instanta- 
neous water use efficiency (WUEins, 23.97 and 78.08\%), but increased leaf temperature (LT, by 12.9 and 17.88\%) and intrinsic water use efficiency (WUEint, 189.31 and 50.97\%) (Figure 2a-f). Contrariwise, impressively improved $P_{n}$ (by 447.91 and $1964.28 \%$ ), $g_{s}(262.79$ and $1094.64 \%), E(151.89$ and $258.76 \%)$, WUEint (50.39 and 74.79\%), and WUEins (119.29 and 476.781\%), and decreased LT (16.01 and 28.23\%) were found in 'S1 + Eth' and 'S2 + Eth' plants, respectively, relative to those values found in the corresponding 'S1' and 'S2' plants (Figure 2a-f). Additionally, as compared with the 'Control' plants, 'Eth' plants displayed enhancements in $P_{n}$ and $E$ by 22.98 and $36.05 \%$, respectively, but a decline in LT by $10.70 \%$ (Figure 2a,c,d).
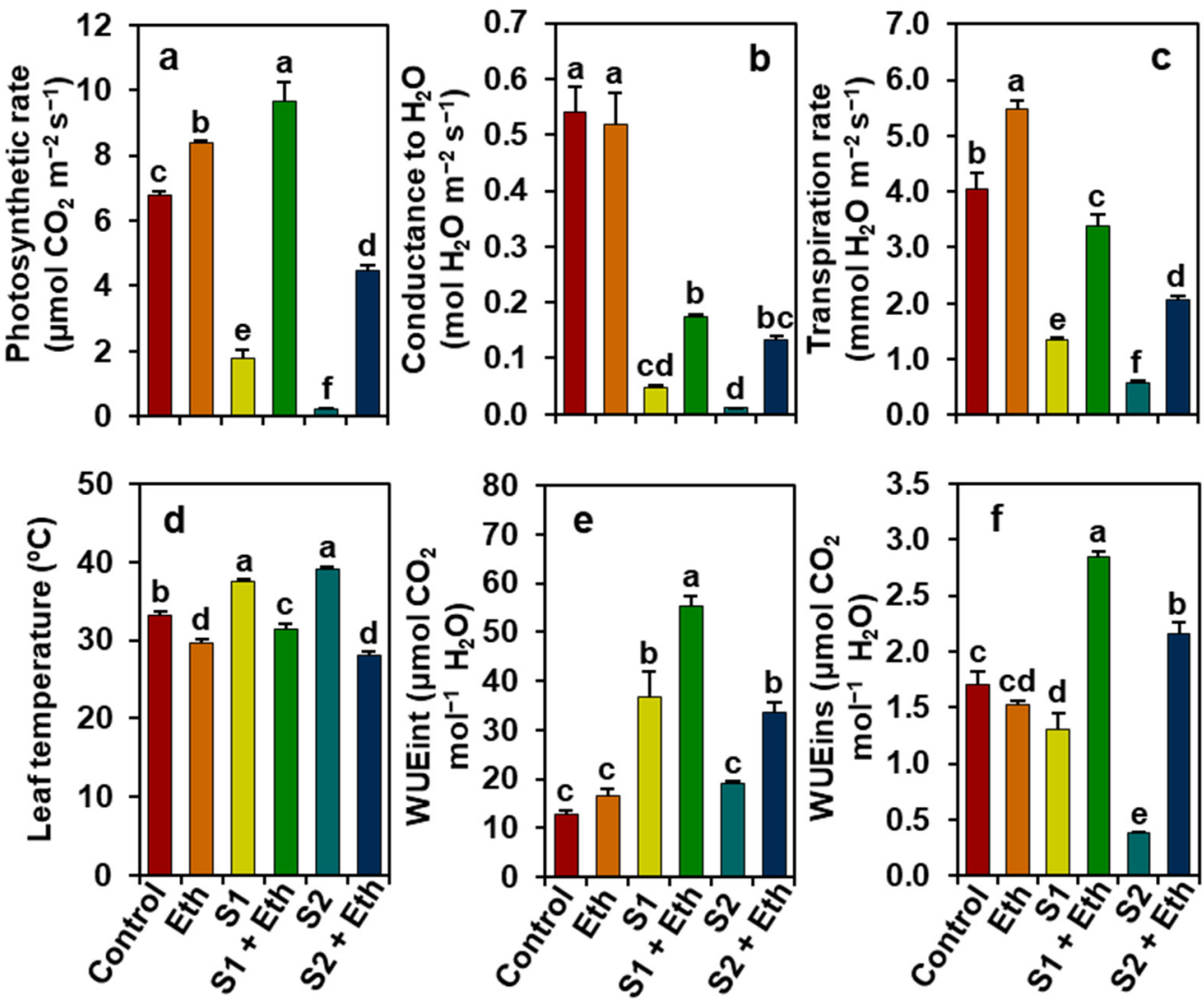

Figure 2. Effects of foliar-sprayed ethanol on (a) photosynthetic rate $\left(P_{n}\right),(\mathbf{b})$ stomatal conductance to $\mathrm{H}_{2} \mathrm{O}\left(g_{s}\right)$, (c) transpiration rate $(E)$, (d) leaf temperature (LT), (e) intrinsic water use efficiency (WUEint), and (f) instantaneous water use efficiency (WUEins) in the leaves of soybean plants exposed to a gradient of salinity for 7 days. The statistically significant differences $(p<0.05)$ among various treatments are shown by different letters following the least significant difference (LSD) test. Eth, $20 \mathrm{mM}$ ethanol; S1, $8 \mathrm{dS} \mathrm{m}^{-1}$; $\mathrm{S} 1+$ Eth, $8 \mathrm{dS} \mathrm{m}^{-1}+20 \mathrm{mM}$ ethanol; S2, $16 \mathrm{dS} \mathrm{m}^{-1}$; S2 + Eth, $16 \mathrm{dS} \mathrm{m}^{-1}+20 \mathrm{mM}$ ethanol.

\subsection{Ethanol Safeguards Photosynthetic Pigments in Salt-Stressed Soybean Plants}

'S1' and 'S2' plants, respectively, exhibited notable reductions in the levels of Chl $a$ (by 42.58 and $68.10 \%), \mathrm{Chl} b$ (48.53 and $72.82 \%)$, total Chls (44.34 and $69.54 \%)$, and carotenoids (53.16 and 64.40\%), when compared with those of the 'Control' plants (Figure 3a-d). By contrast, 'S1 + Eth' and 'S2 + Eth' plants, respectively, showed remarkable increases in the levels of Chl $a$ (by 67.41 and 131.36\%), Chl $b$ (111.26 and 185.85\%), total Chls (78.95 and $146.15 \%$ ), and carotenoids (97.10 and $123.92 \%)$, corresponding to 'S1' and 'S2' plants (Figure 3a-d). 

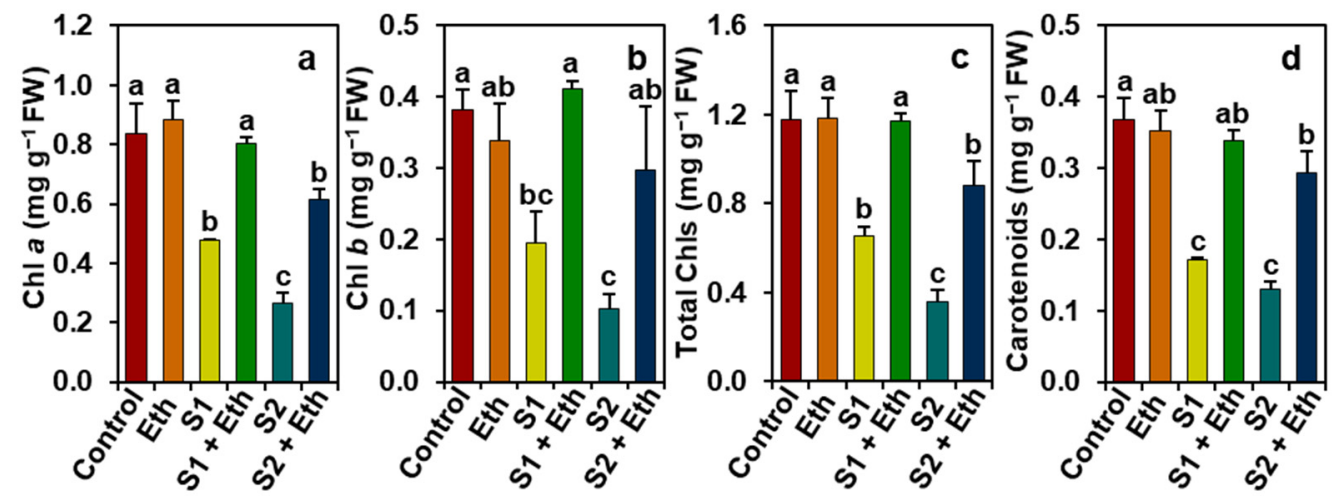

Figure 3. Effects of foliar-sprayed ethanol on the levels of (a) Chl $a$, (b) Chl b, (c) total Chls, and (d) carotenoids in leaves of soybean plants exposed to a gradient of salinity for 7 days. The statistically significant differences $(p<0.05)$ among various treatments are shown by different letters following the least significant difference (LSD) test. Chl, Chlorophyll; FW, fresh weight; Eth, 20 mM ethanol; S1, $8 \mathrm{dS} \mathrm{m}^{-1}$; S1 + Eth, $8 \mathrm{dS} \mathrm{m}{ }^{-1}+20 \mathrm{mM}$ ethanol; S2, $16 \mathrm{dS} \mathrm{m}^{-1}$; S2 + Eth, $16 \mathrm{dS} \mathrm{m}^{-1}+20 \mathrm{mM}$ ethanol.

\subsection{Ethanol Reduces Oxidative Damage in Soybean Plants under Salt Stress}

Relative to the 'Control' plant leaves, staining of the leaves of 'S1' and 'S2' plants with nitro blue tetrazolium (NBT) for superoxide $\left(\mathrm{O}_{2}{ }^{\bullet-}\right)$ and 3,3'-diaminobenzidine (DAB) for hydrogen peroxide $\left(\mathrm{H}_{2} \mathrm{O}_{2}\right)$ resulted in the development of more deep blue spots and dark brown spots, respectively (Figure 4a,b). By comparison, 'S1 + Eth' and 'S2 + Eth' plant leaves showed a notable reduction in the accumulation of $\mathrm{O}_{2}{ }^{\bullet-}$ and $\mathrm{H}_{2} \mathrm{O}_{2}$, when contrasted with the corresponding 'S1' and 'S2' plant leaves (Figure 4a,b). More specifically, the leaves of 'S1' and 'S2' plants exhibited significant enhancements in the levels of $\mathrm{H}_{2} \mathrm{O}_{2}$ by 1016.50 and $1621.93 \%$, malondialdehyde (MDA) by 154.92 and $277.87 \%$, and electrolyte leakage (EL) by 361.44 and $823.76 \%$, respectively, as compared with the level recorded in the 'Control' plant leaves (Figure 4c-e). In contrast, reduced contents of $\mathrm{H}_{2} \mathrm{O}_{2}$ (by 65.95 and $67.82 \%)$, MDA (66.67 and 65.40\%), and EL (57.26 and 46.93\%) were found in 'S1 + Eth' and 'S2 + Eth' plant leaves, respectively, when compared with the corresponding values in 'S1' and 'S2' plant leaves (Figure 4c-e).
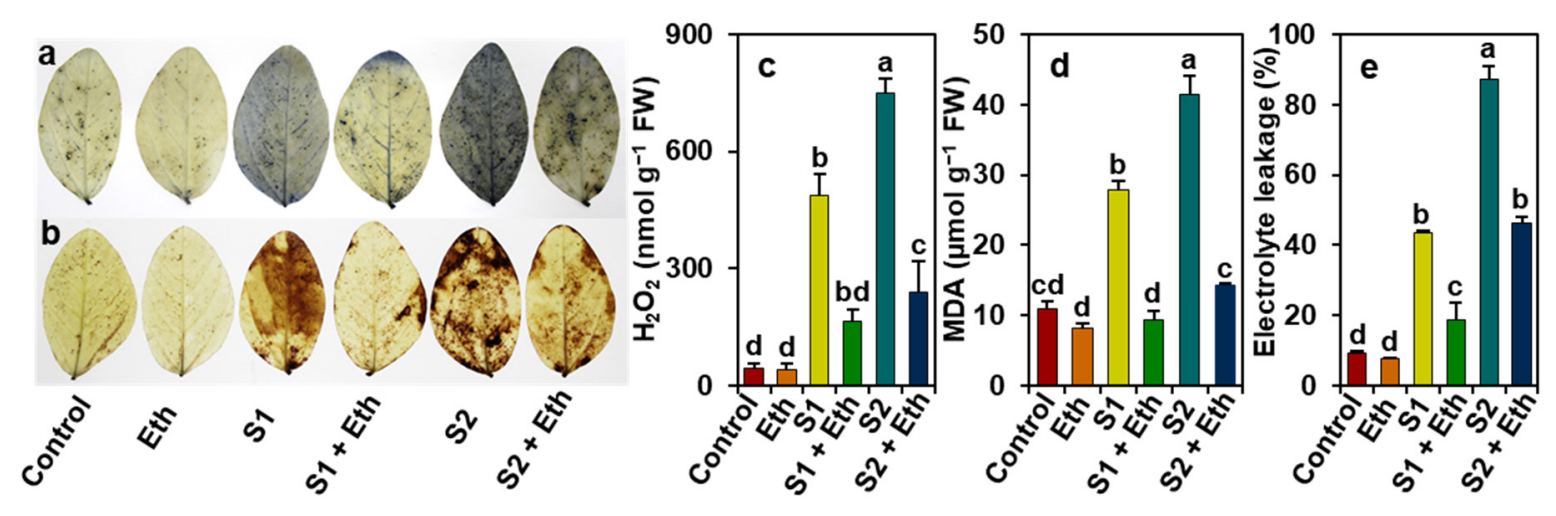

Figure 4. Effects of foliar-sprayed ethanol on reactive oxygen species accumulations in leaves of soybean plants exposed to a gradient of salinity for 7 days. (a) Superoxide $\left(\mathrm{O}_{2}{ }^{\bullet-}\right)$ and (b) hydrogen peroxide $\left(\mathrm{H}_{2} \mathrm{O}_{2}\right)$ accumulations were stained with NBT and DAB solutions, respectively. Levels of (c) $\mathrm{H}_{2} \mathrm{O}_{2}$, (d) malondialdehyde (MDA), and (e) electrolyte leakage (EL) in the leaves of soybean plants. The statistically significant differences $(p<0.05)$ among various treatments are shown by different letters following the least significant difference (LSD) test. FW, fresh weight; Eth, $20 \mathrm{mM}$ ethanol; S1, $8 \mathrm{dS} \mathrm{m}^{-1}$; S1 + Eth, $8 \mathrm{dS} \mathrm{m}^{-1}+20 \mathrm{mM}$ ethanol; S2, $16 \mathrm{dS} \mathrm{m}^{-1}$; S2 + Eth, $16 \mathrm{dS} \mathrm{m}^{-1}+20 \mathrm{mM}$ ethanol. NBT; nitroblue tetrazolium; DAB; 3,3'-diaminobenzidine. 


\subsection{Ethanol Improves Antioxidant Defense Responses in Salt-Stressed Soybean Plants}

Relative to the 'Control' plants, 'S1' and 'S2' plants showed enhancements in the activities of APX (by 148.46\% in 'S2'), peroxidase (POD, 46.27\% in 'S2'), and GST (47.71 and $157.84 \%$, respectively) (Figure 5b-d). However, the activities of CAT decreased by 21.45 and $33.20 \%$ in 'S1' and 'S2' plants, respectively, compared with that found in the 'Control' plants (Figure 5a). In contrast, notably improved activities of CAT (by 73.33 and $83.55 \%$ ), APX (114.66 and 20.73\%), POD (140.38 and 16.83\%), and GST (58.34 and 56.37\%) were observed in 'S1 + Eth' and 'S2 + Eth' plants, respectively, in contrast to those observed in 'S1' and 'S2' plants (Figure 5a-d). In addition, 'Eth' plants also showed significant enhancements in the activities of CAT, APX, POD, and GST by 73.89, 121.14, 17.41, and 104.25\%, respectively, relative to the 'Control' plants (Figure 5a-d).
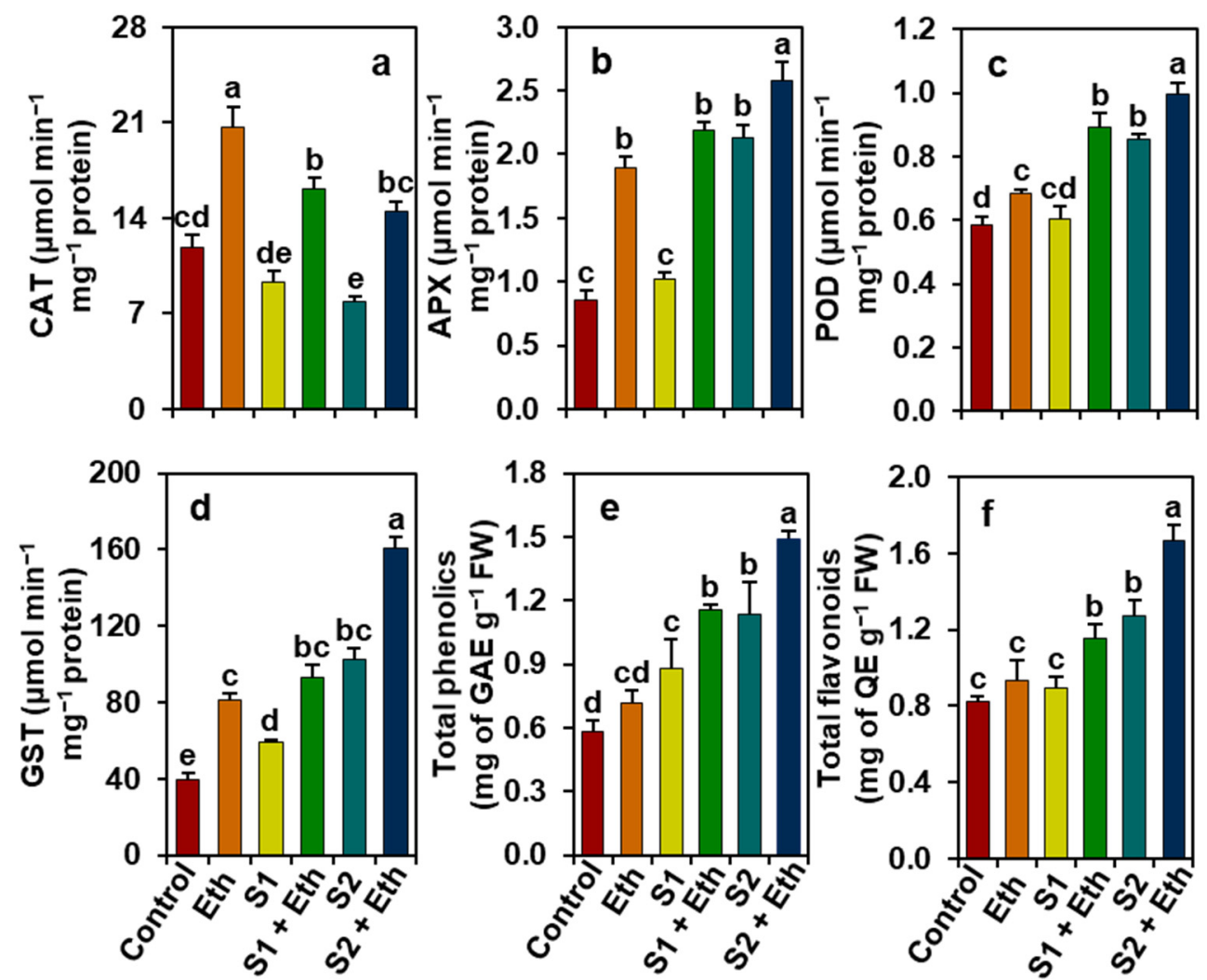

Figure 5. Effects of foliar-sprayed ethanol on (a) catalase (CAT), (b) ascorbate peroxidase (APX), (c) peroxidase (POD), and (d) glutathione $S$-transferase (GST) activities, and the levels of (e) total phenolics and (f) total flavonoids in leaves of soybean plants exposed to a gradient of salinity for 7 days. The statistically significant differences $(p<0.05)$ among various treatments are shown by different letters following the least significant difference (LSD) test. FW, fresh weight; Eth, $20 \mathrm{mM}$ ethanol; S1, $8 \mathrm{dS} \mathrm{m}^{-1}$; S1 + Eth, $8 \mathrm{dS} \mathrm{m}^{-1}+20 \mathrm{mM}$ ethanol; S2, $16 \mathrm{dS} \mathrm{m} \mathrm{m}^{-1}$; S2 + Eth, $16 \mathrm{dS} \mathrm{m} \mathrm{m}^{-1}+20 \mathrm{mM}$ ethanol; GAE, gallic acid equivalent; $\mathrm{QE}$, quercetin equivalent.

On the other hand, 'S1' and 'S2' plants showed noteworthy augmentations in the levels of total phenolics (by 50.73 and $94.63 \%$, respectively) and total flavonoids (55.22\% in 'S2'), when compared with the 'Control' plants (Figure 5e,f). By comparison, further enhancements in the content of total phenolics (by 31.36 and 30.87\%) and total flavonoids (29.97 and 30.86\%) were found in 'S1 + Eth' and 'S2 + Eth' plants, respectively, unlike in those observed in 'S1' and 'S2' plants (Figure 5e,f).

\subsection{Ethanol Enhances the Levels of Osmoprotectants in Salt-Stressed Soybean Plants}

In relation to the 'Control' plants, 'S1' and 'S2' plants showed lower levels of leaf relative water content (RWC, by 38.15 and 59.33\%, respectively) and water-soluble protein 
(27.09\% in 'S2'), but higher levels of Pro (by 3039.72 and $1335.32 \%$, respectively), total free amino acids (116.25\% in 'S2'), and total soluble sugars (252.53 and $299.07 \%$, respectively) (Table 1). In contrast, 'S1 + Eth' and 'S2 + Eth' plants, respectively, displayed significantly higher levels of leaf RWC (by 33.03 and 90.78\%), Pro (50.49 and 91.44\%), and total free amino acids (58.96 and 33.10\%), but lower levels of water-soluble protein (by 53.92 and $35.02 \%$ ) and total soluble sugars (50.69 and 44.72\%) than the corresponding salt-stressed plants only (Table 1). Additionally, the levels of water-soluble proteins in 'Eth' plants decreased by $42.85 \%$ when compared with the 'Control' plants (Table 1 ).

Table 1. Effects of exogenous ethanol in modulation of the levels of water content, free amino acids, proline, water-soluble proteins, and soluble sugars in the leaves of soybean plants exposed to a gradient of salinity for 7 days.

\begin{tabular}{|c|c|c|c|c|c|}
\hline Treatment & $\begin{array}{c}\text { Leaf Relative } \\
\text { Water Content (\%) }\end{array}$ & $\begin{array}{l}\text { Total Free Amino } \\
\text { Acids }\left(\mu \mathrm{g} \mathrm{g}^{-1} \text { FW) }\right.\end{array}$ & $\begin{array}{c}\text { Proline } \\
\left(\mu \mathrm{mol} \mathrm{g}^{-1} \mathrm{FW}\right)\end{array}$ & $\begin{array}{c}\text { Water-Soluble } \\
\text { Proteins (mg g }{ }^{-1} \text { FW) }\end{array}$ & $\begin{array}{c}\text { Total Soluble } \\
\left.\text { Sugars (mg g }{ }^{-1} \mathrm{FW}\right)\end{array}$ \\
\hline Control & $86.41 \pm 3.59^{a b}$ & $45.54 \pm 1.00^{c}$ & $0.63 \pm 0.04^{\mathrm{e}}$ & $7.06 \pm 0.33^{b}$ & $9.93 \pm 0.11^{\mathrm{c}}$ \\
\hline Eth & $88.92 \pm 0.88^{a}$ & $43.95 \pm 0.21^{c}$ & $0.74 \pm 0.05^{\mathrm{e}}$ & $4.04 \pm 0.10^{\mathrm{d}}$ & $8.32 \pm 0.65^{c}$ \\
\hline S1 & $53.44 \pm 4.96^{\mathrm{d}}$ & $62.04 \pm 0.52^{c}$ & $20.00 \pm 0.87^{b}$ & $8.58 \pm 0.09^{a}$ & $34.99 \pm 2.64^{a}$ \\
\hline S1 + Eth & $71.10 \pm 1.75^{b c}$ & $98.60 \pm 4.24^{b}$ & $30.10 \pm 0.30^{a}$ & $3.95 \pm 0.07^{\mathrm{d}}$ & $17.26 \pm 1.25^{b}$ \\
\hline $\mathrm{S} 2$ & $35.14 \pm 9.24^{\mathrm{e}}$ & $98.48 \pm 17.91^{b}$ & $9.15 \pm 0.81^{\mathrm{d}}$ & $5.15 \pm 0.08^{c}$ & $39.61 \pm 3.17^{\mathrm{a}}$ \\
\hline $\mathrm{S} 2+$ Eth & $67.04 \pm 1.26^{\mathrm{cd}}$ & $131.08 \pm 1.29^{a}$ & $17.50 \pm 1.07^{c}$ & $3.35 \pm 0.12^{\mathrm{e}}$ & $21.90 \pm 1.99^{b}$ \\
\hline
\end{tabular}

\subsection{Ethanol Maintains Mineral Balance in Salt-Stressed Soybean Plants}

Relative to 'Control' plants' roots, 'S1' and 'S2' plants' roots, respectively, showed a significant increase in $\mathrm{Na}^{+}$content (by 239.61 and 350.57\%) and a decrease in potassium ion $\left(\mathrm{K}^{+}\right)$content $\left(40.36\right.$ and $57.18 \%$ ). Consequently, notable reductions in root $\mathrm{K}^{+} / \mathrm{Na}^{+}$ratios (by 82.56 and $90.56 \%$, respectively) were observed in 'S1' and 'S2' plants, relative to the 'Control' plants (Figure 6a-c). By comparison, 'S1 + Eth' and 'S2 + Eth' plants' roots, respectively, exhibited a reduced level of $\mathrm{Na}^{+}$(by 34.78 and 38.87\%) and an augmented level of $\mathrm{K}^{+}(62.61$ and $151.85 \%$ ), resulting in a higher $\mathrm{K}^{+} / \mathrm{Na}^{+}$ratio (by 150.49 and $312.58 \%$ ), when compared with the corresponding values recorded in the roots of 'S1' and 'S2' plants (Figure 6a-c). However, the levels of magnesium ions $\left(\mathrm{Mg}^{2+}\right)$ remained comparable between 'S1' and 'S2', and 'Control' plants (Figure 6d). However, 'S1 + Eth' and 'S2 + Eth' plants' roots displayed an increase in $\mathrm{Mg}^{2+}$ levels by 6.42 and $8.77 \%$, respectively, in comparison to the corresponding 'S1' and 'S2' plants (Figure 6d). In 'Eth' plants, the root $\mathrm{Mg}^{2+}$ content significantly increased by $5.66 \%$ compared to that in the 'Control' plants; however, $\mathrm{Na}^{+}$ and $\mathrm{K}^{+}$levels remained comparable between 'Eth' and 'Control' plants (Figure 6a,b,d). Interestingly, in 'Eth' plants, the $\mathrm{K}^{+} / \mathrm{Na}^{+}$ratio in the roots increased by $31.02 \%$ compared to that in the 'Control' plants (Figure 6c).

The leaves of 'S1' and 'S2' plants, respectively, exhibited a significant upsurge in $\mathrm{Na}^{+}$ content (by 196.11 and $270.43 \%$ ) and a decline in $\mathrm{K}^{+}$content (by 40.72 and $73.19 \%$ ), resulting in a lower $\mathrm{K}^{+} / \mathrm{Na}^{+}$ratio (by 80.75 and $93.01 \%$ ), as compared with the observed values in the 'Control' plants (Figure 6a-c). In contrast, ethanol supplementation attenuated $\mathrm{Na}^{+}$content (by 41.89 and 35.63\%) and enhanced $\mathrm{K}^{+}$content (122.39 and 399.69\%) in the leaves of 'S1 + Eth' and 'S2 + Eth' plants, respectively, resulting in an increase in the $\mathrm{K}^{+} / \mathrm{Na}^{+}$ratio by 283.01 and $673.66 \%$, respectively, when compared with the 'Control' plants (Figure $6 \mathrm{a}-\mathrm{c}$ ). In addition, 'S1 + Eth' and 'S2 + Eth' plants also showed an enhanced $\mathrm{Mg}^{2+}$ content in the leaves by 10.31 and $14.23 \%$, respectively, in relation to the 'Control' plants (Figure 6d). Likewise, in comparison with the 'Control' plants, 'Eth' plants showed a 
reduced accumulation of $\mathrm{Na}^{+}$by $31.83 \%$, an increased accumulation of $\mathrm{K}^{+}$by $18.87 \%$, and consequently, a higher $\mathrm{K}^{+} / \mathrm{Na}^{+}$ratio by $73.10 \%$ in the leaves (Figure $6 \mathrm{a}-\mathrm{c}$ ).

Furthermore, compared with those of the 'Control' plants, ion ratios within different plant organs showed that 'S1' and 'S2' plants displayed a reduced leaf/root ratio of $\mathrm{Na}^{+}$ content (by $18.87 \%$ in 'S2') and $\mathrm{K}^{+}$content (by 35.55 in 'S2'), while in the case of $\mathrm{Mg}^{2+}$, the reduction was not significant for the same treatments (Figure 6e). Interestingly, in 'S1 + Eth' and 'S2 + Eth' plants, a remarkably reduced leaf/root ratio of $\mathrm{Na}^{+}$content (by $10.18 \%$ in 'S1') and an increased leaf/root ratio of $\mathrm{K}^{+}$content (38.29 and $93.58 \%$, respectively) was observed, when compared with those of corresponding values found in 'S1' and 'S2' plants (Figure 6e). Additionally, in comparison with the 'Control' plants, 'Eth' plants also displayed a lower leaf/root ratio of $\mathrm{Na}^{+}$content (by 32.27\%) (Figure 6e).

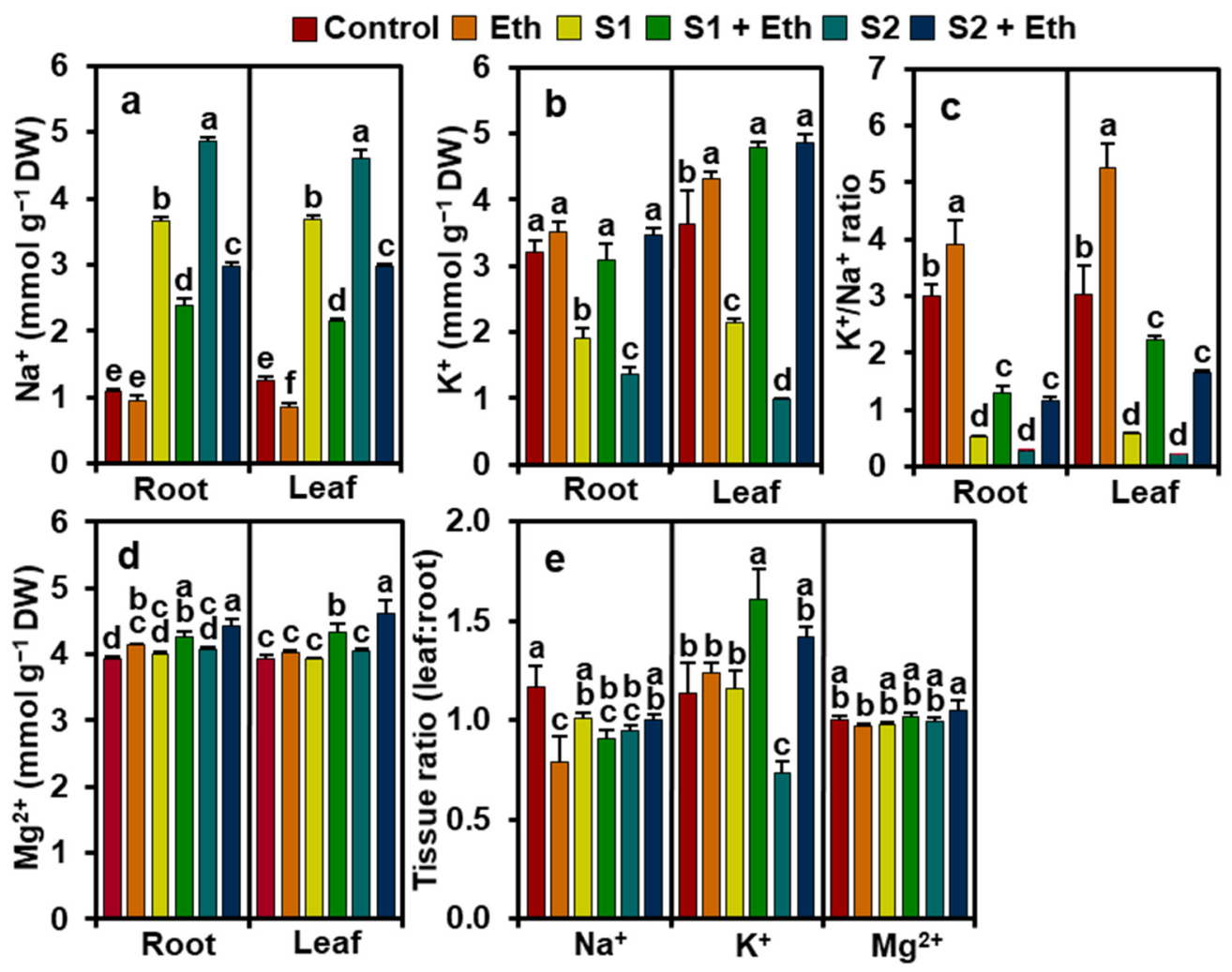

Figure 6. The content of different nutrients and tissue mineral ratios (leaf: root) among different parts of soybean plants exposed to a gradient of salinity for 7 days. Levels of (a) $\mathrm{Na}^{+}$, (b) $\mathrm{K}^{+}$, (c) $\mathrm{K}^{+} / \mathrm{Na}^{+}$ ratio, and (d) $\mathrm{Mg}^{2+}$, and tissue ratios of $(\mathbf{e}) \mathrm{Na}^{+}, \mathrm{K}^{+}$, and $\mathrm{Mg}^{2+}$ for leaf/root of soybean plants. Values (means \pm SEs) of each treatment were attained from six biological replications $(n=6)$. The statistically significant differences $(p<0.05)$ among various treatments are shown by different letters following the least significant difference (LSD) test. DW, dry weight; Eth, $20 \mathrm{mM}$ ethanol; $\mathrm{S1}, 8 \mathrm{dS} \mathrm{m}^{-1}$; $\mathrm{S} 1+$ Eth, $8 \mathrm{dS} \mathrm{m}{ }^{-1}+20 \mathrm{mM}$ ethanol; S2, $16 \mathrm{dS} \mathrm{m}^{-1} ; \mathrm{S} 2+$ Eth, $16 \mathrm{dS} \mathrm{m}^{-1}+20 \mathrm{mM}$ ethanol.

\subsection{Clustering Heatmap-Based Data Visualization under Different Treatments and Treatment-Parameter Association by Principal Component Analysis}

A heatmap was generated to visualize the performance of different parameters under different treatment conditions using color intensity, and the parameters were further grouped into four different clusters using the hierarchical clustering method (Figure 7a). When compared with the 'Control' plants, the parameters of cluster-A revealed a declining trend in 'S1' and 'S2' plants. Interestingly, 'S1 + Eth,' 'S2 + Eth,' and 'Eth' plants displayed a reverse trend than those of 'S1' and 'S2' plants for cluster-A parameters, with the exception of Chl $b$, carotenoids, and WUEins in 'Eth' plants (Figure 7a). In comparison to the 'Control' plants, most of the variables in cluster-B exhibited an upward trend under both levels of salt stress. However, there were further escalations in the levels of these parameters in 'S1 + Eth,' 
'S2 + Eth,' and 'Eth' plants, with exceptions in the cases of $\mathrm{Mg}^{2+}$ for leaf/root and total free amino acids in 'Eth' plants (Figure 7a). When compared with the respective 'Control' plants, parameters of cluster-C exhibited a changeable trend under saline conditions; nevertheless, all of the features of this cluster declined upon ethanol treatment (Figure 7a). In comparison with the 'control' plants, parameters of cluster-D displayed an increasing tendency under stress conditions; however, foliar ethanol application reverses the trend in both salt-stressed and non-stressed plants (Figure 7a). Subsequently, to find out the association between different treatments and variables, principal component analysis (PCA) was carried out (Figure $7 \mathrm{~b})$. The PC1 (57.92\%) and PC2 (26.52\%) accounted for the majority of the variability and collectively explained $84.44 \%$ of the variability. Notably, parameters of cluster-A and cluster-B were found to have a close association with 'S1 + Eth' and 'S2 + Eth' treatments, while cluster-B and -C variables were found to be closely related to 'S1' and 'S2' treatments (Figure 7a,b). Nonetheless, 'Eth' plants showed a close relationship with cluster-A and -B variables instead of cluster-C and -D variables (Figure 7a,b).

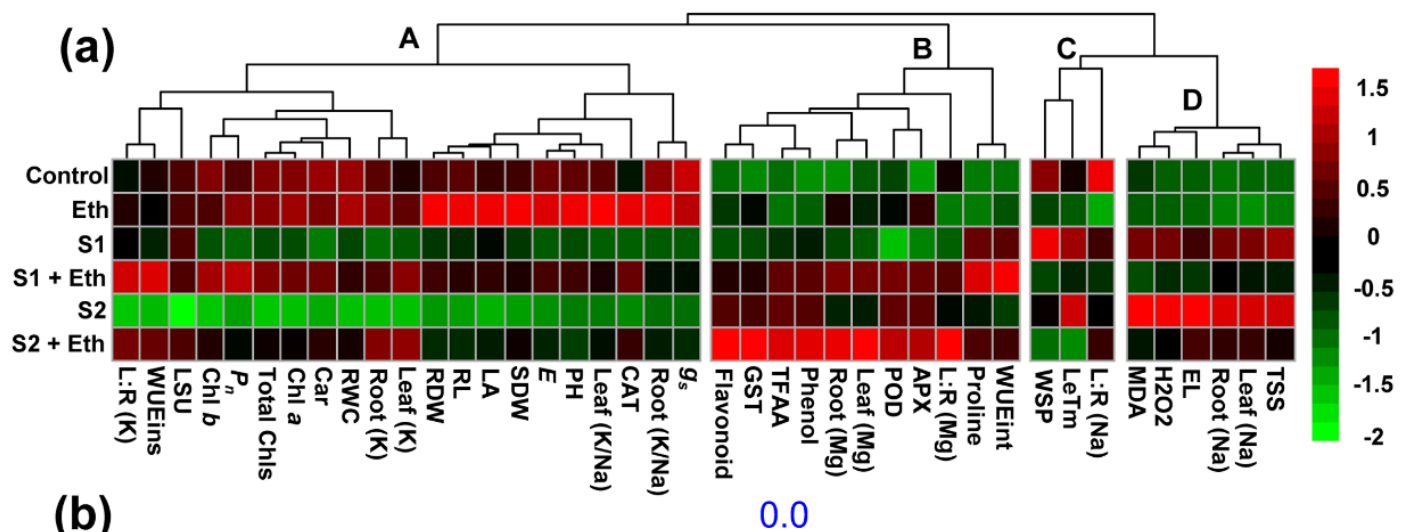

(b)

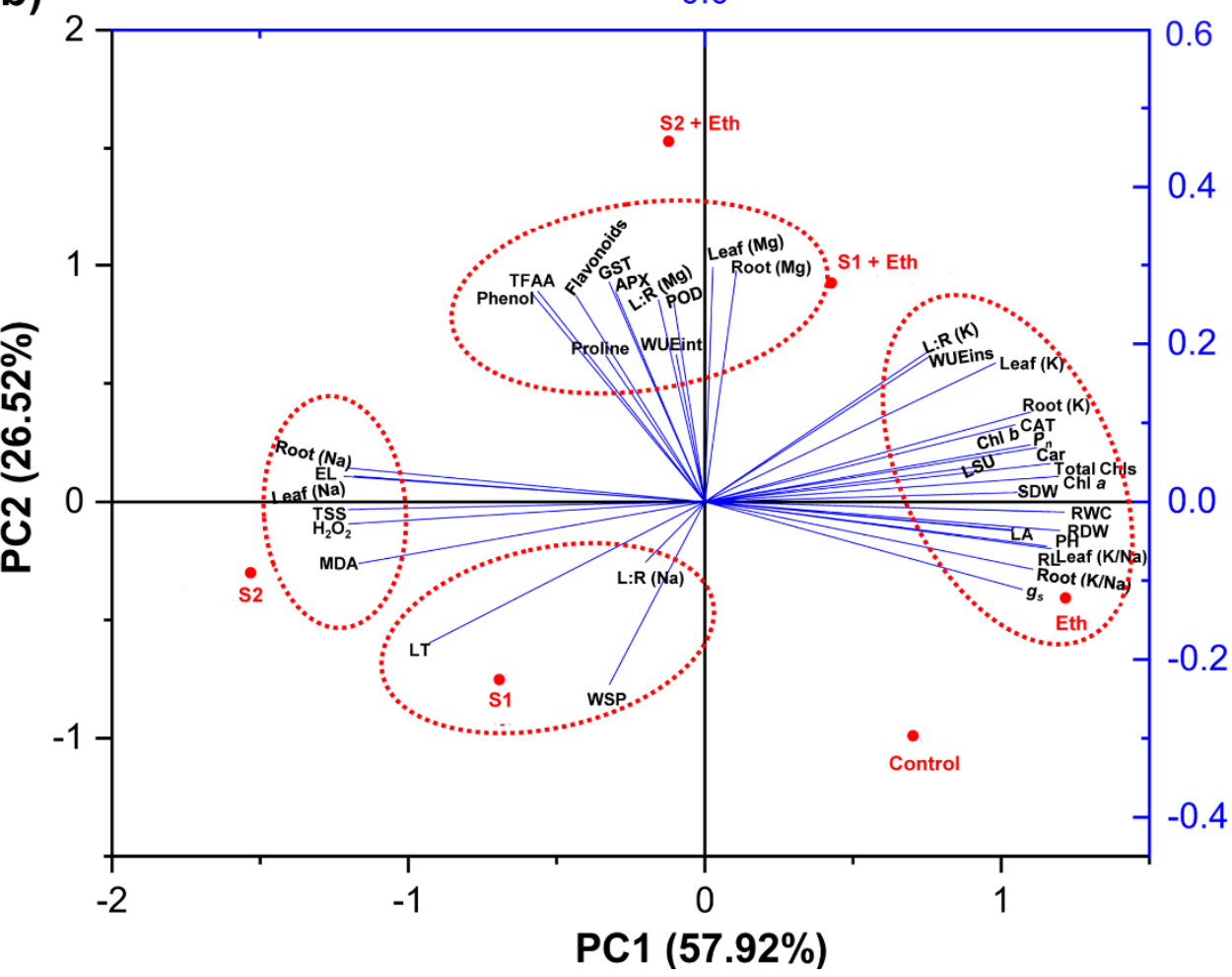

Figure 7. (a) Clustering heatmap visualizing different parameters under different treatments at a glance. Normalized mean values of different parameters were used to prepare the heatmap. The parameters were grouped into four distinct clusters. (b) Principal component analysis (PCA) represents the relationship among the different treatments and parameters. The biplot was created 
with the first two components (PC1 and PC2) that collectively explains $84.44 \%$ of the variability among the datasets. The vector lines of the biplot display positive or negative associations of different morpho-physiological and biochemical parameters with different treatments. The angle value between the parameter and treatment specifies the intensity of association between treatment and subsequent parameter, where a small angle indicates a weak association, and a large angle indicates a strong association. The parameters included RDW (root dry weight), RL (root length), LA (total leaf area per trifoliate), Leaf (K/Na) (leaf $\mathrm{K}^{+}$and $\mathrm{Na}^{+}$ratio), SDW (shoot dry weight), $E$ (transpiration rate), $\mathrm{PH}$ (plant height), CAT (catalase), Total Chls (total chlorophylls), Chl $a$ (chlorophyll $a$ ), Chl $b$ (chlorophyll $b$ ), Car (carotenoids), RWC (relative water content), $P_{n}$ (photosynthetic rate), $g_{s}$ (conductance to $\mathrm{H}_{2} \mathrm{O}$ ), Leaf (K) (leaf $\mathrm{K}^{+}$content), LSU (leaf succulence), WUEins (instantaneous water-use efficiency), Root $(\mathrm{Mg})$ (root $\mathrm{Mg}^{2+}$ content), Leaf $(\mathrm{Mg})$ (leaf $\mathrm{Mg}^{2+}$ content), L:R (K) (leaf and root ratio of $\mathrm{K}^{+}$content), $\mathrm{L}: \mathrm{R}(\mathrm{Mg})$ (leaf and root ratio of $\mathrm{Mg}^{2+}$ content), Root (Na) (root $\mathrm{Na}^{+}$ content), APX (ascorbate peroxidase), TFAA (total free amino acids), Phenol (total phenolics), POD (peroxidase), Flavonoids (total flavonoids), GST (glutathione $S$-transferase), Pro (proline), WUEint (intrinsic water-use efficiency), WSP (water-soluble protein), LT (leaf temperature), Root (K/Na) (root $\mathrm{K}^{+}$and $\mathrm{Na}^{+}$ratio), $\operatorname{Root}(\mathrm{K})$ (root $\mathrm{K}^{+}$content), MDA (malondialdehyde), $\mathrm{H}_{2} \mathrm{O}_{2}$ (hydrogen peroxide), EL (electrolyte leakage), L:R (Na) (leaf and root ratio of $\mathrm{Na}^{+}$content), Leaf ( $\mathrm{Na}$ ) (leaf $\mathrm{Na}^{+}$content), and TSS (total soluble sugars). Eth, ethanol; S1, $8 \mathrm{dS} \mathrm{m}^{-1}$; $\mathrm{S} 1+$ Eth, $8 \mathrm{dS} \mathrm{m} \mathrm{m}^{-1}+20 \mathrm{mM}$ ethanol; S2, $16 \mathrm{dS} \mathrm{m}^{-1} ; \mathrm{S} 2+$ Eth, $16 \mathrm{dS} \mathrm{m}^{-1}+20 \mathrm{mM}$ ethanol.

\section{Discussion}

Soil salinity has emerged as a severe environmental problem that has a variety of detrimental effects on plant growth and development $[6,21]$. In this study, we found that soybean plants subjected to salt stress displayed a distortion of morphological features, including the wilting and yellowing of leaves, as well as reductions in root length, shoot height, shoot DW and root DW, leaf area per trifoliate, and leaf succulence, when compared with control conditions (Figure $1 \mathrm{a}-\mathrm{j}$ ). These results were corroborated with previous findings in other salt-stressed legume crops, such as lentil (Lens culinaris) and mung bean (Vigna radiata) [6,22]. Conversely, the foliar application of ethanol to salt-stressed plants resulted in decreased canopy wilting and yellowing, as well as restored growth rate and biomass production, thereby playing a decisive role in alleviating salt-mediated deleterious effects in soybean plants (Figure 1a-j). Our findings were further supported by PCA, which revealed that salt-stressed soybean plants supplemented with ethanol had a less negative interaction with growth features than ethanol-devoid stressed plants (Figure 7b). The positive regulatory role of ethanol in enhancing plant growth performance has also been reported by Rowe et al. [23], Yavarpanah et al. [24], and Nguyen et al. [20].

The impairment of phenotype and reduction in growth and biomass might be a consequence of abnormal photosynthesis (Figure 2a), which could be mediated by the salt-induced destruction of photosynthetic pigments as a result of an enhancement of ROS production $[25,26]$. Our results showed that salt-stressed soybean plants had substantially lower levels of photosynthetic pigments (Figure $3 a-d$ ), whereas the application of exogenous ethanol to salt-exposed plants retained photosynthetic pigment levels (e.g., Chls and carotenoids) and net photosynthetic rates, compared with salt-stressed plants only (Figures $2 \mathrm{a}$ and $3 \mathrm{a}-\mathrm{d}$ ). These findings suggest that ethanol may have a positive role in the prevention and/or delaying of the destruction of photosynthetic pigments (Figure 3a-d), resulting in an enhancement of photosynthesis capacity of soybean plants under salinity (Figure 2a), which was supported by the findings of Nguyen et al. [20]. In addition, the improved photosynthetic rate in ethanol-sprayed salt-stressed soybean plants might be associated with a greater leaf area per trifoliate (Figure 1i), which ensures the maximum light interception capacity that ultimately boosts the photosynthetic rate in plants [27-29]. It is worth noting that ethanol enabled salt-stressed soybean plants to retain greater photosynthesis by boosting their WUE under the physiological drought circumstances, as evidenced 
by higher levels of WUEint and WUEins, leading to more biomass gain than salt-stressed plants only. Interestingly, higher transpiration rates in ethanol-supplemented salt-stressed plants contributed to the maintenance of leaf cooling, as evidenced by their lower LT than the plants stressed with salt only (Figure 3c,d). Our PCA analysis also provided compelling evidence that the beneficial effects of external ethanol in salt-stressed soybean plants were positively correlated with improved photosynthesis and WUE (Figure 7b).

Our findings also revealed that salt stress led to an increased accumulation of ROS products, including $\mathrm{O}_{2}{ }^{\bullet-}$ and $\mathrm{H}_{2} \mathrm{O}_{2}$ (Figure $4 \mathrm{a}$, b, respectively). These results together with high levels of MDA and EL indicated a greater degree of membrane damage in salt-stressed soybean plants (Figure 4a-e). The addition of exogenous ethanol, on the other hand, helped minimize the burden of ROS-mediated oxidative damage and provided protection against cell membrane damage, as evidenced by diminished levels of ROS, MDA, and EL in saltstressed soybean leaf tissues (Figure 4a-e). In line with our findings, PCA analysis also demonstrated a negative interaction between the treatments of ethanol-sprayed soybean plants and the levels of $\mathrm{H}_{2} \mathrm{O}_{2}, \mathrm{MDA}$, and EL; however, a positive association was observed in water-sprayed salt-treated plants (Figure $7 \mathrm{~b}$ ).

Plants have evolved a robust antioxidant defense mechanism that includes both enzymatic and nonenzymatic antioxidants to combat ROS-induced oxidative damage under salt stress $[2,30]$. In the current study, we determined the activities of several key enzymes and the levels of nonenzymatic antioxidants such as total phenolics and flavonoids to identify the effective roles of ethanol in oxidative stress mitigation. Our findings showed that water-sprayed salt-exposed plants enhanced the activity of APX, GST, and POD; however, the activity of CAT was observed to be decreased (Figure 5a-d). The ethanol-supplemented salt-stressed soybean plants, on the other hand, further increased the activities of APX, POD, GST, and CAT, compared with salt-stressed plants alone (Figure 5a-d). It is plausible that the elevated activities of CAT, APX, and POD greatly contributed to the reduction in oxidative damage by detoxifying $\mathrm{H}_{2} \mathrm{O}_{2}$ in the leaves of ethanol-added salt-stressed soybean plants. Furthermore, GST is an important enzymatic player for activating glutathionedependent peroxide-detoxification system, which ensures greater protection against lipid hydroperoxides, reactive aldehydes and ketones, and organic peroxides produced from the effects of high salinity [30]. In previous findings, transcriptome analyses of ethanol-treated salt-exposed Arabidopsis plants revealed the upregulation of several genes involved in the regulation of ROS homeostasis under salinity, including AtAPX1, AtAPX2, AtGSTU4, and AtGSTU19 [20]. Our findings further demonstrated that ethanol addition boosted the levels of nonenzymatic antioxidants, including total phenolics and flavonoids, in salt-stressed soybean plants (Figure 5e,f), which might have played a crucial role in safeguarding the cell membrane from oxidative damage by scavenging toxic ROS during salinity stress [6,31-33]. Our results were supported by the PCA analysis, which demonstrated a substantial positive correlation between the treatments of ethanol-supplemented salt-stressed plants and the activities and/or levels of enzymatic and nonenzymatic antioxidants (Figure 7b).

Plants produce a wide array of osmoprotectants to support osmotic balance under salinity stress [34]. The results of the current study disclosed that excessive salt stress resulted in a significant accumulation of free amino acids, Pro, and total soluble sugars in soybean plants (Table 1). Intriguingly, ethanol treatment of salt-stressed soybean plants further increased the levels of Pro and free amino acids, but reduced the levels of soluble sugars when contrasted with that in salt-stressed plants alone (Table 1). Likewise, the PCA biplot revealed a robust and positive relationship between foliar ethanol application on salt-stressed soybean plants and the levels of the osmoprotectants, Pro and total free amino acids, whereas total soluble sugar exhibited a negative relationship (Figure $7 \mathrm{~b}$ ). The augmented level of Pro in ethanol-supplied salt-stressed soybean plants might help in retaining the water status of the plant, as reflected by higher levels of leaf succulence and RWC (Figure 1j, Table 1). In addition, Pro might provide protection to photosynthetic machineries, cell membranes, and protein functions by scavenging ROS [35-37]. An enhanced accumulation of free amino acids, on the other hand, aided plants in maintaining 
optimum protein synthesis by supplying an adequate supply of amino acids $[27,38,39]$. In accordance with our findings, a positive correlation between improved salt tolerance and the levels of free amino acids and Pro has also been reported in faba bean (Vicia faba) and $V$. radiata $[6,35]$.

Importantly, the poor growth performance of soybean plants under salt stress might be a consequence of an imbalanced nutrient distribution, as manifested by the greater accumulation of toxic $\mathrm{Na}^{+}$and a notable decline in beneficial $\mathrm{K}^{+}$levels (Figures 1 and 6a,b). A salt-induced nutrient imbalance was further evidenced from PCA biplot analysis, which showed the strong and positive associations between salt stress treatments and $\mathrm{Na}^{+}$accumulation in different tissues of soybean plants (Figure $7 \mathrm{~b}$ ). Intriguingly, adding ethanol to salt-treated plants notably enhanced the levels of $\mathrm{K}^{+}$and $\mathrm{Mg}^{2+}$ while abating $\mathrm{Na}^{+}$levels in both leaves and roots (Figure $6 a, b, d$ ). In addition, a lower leaf/root ratio of $\mathrm{Na}^{+}$but a higher leaf/root ratio of $\mathrm{K}^{+}$in ethanol-supplemented salt-stressed soybean plants also indicated a preferential nutrient allocation for maintaining better growth under saline conditions (Figure 6e). Therefore, ethanol-mediated ion homeostasis provided an indirect but strong indication that ethanol played a pivotal role in effective $\mathrm{Na}^{+}$sequestration into the vacuoles, which was further substantiated by increased leaf succulence (Figure $1 j$ ). Plants with succulent features have the ability to dilute absorbed salts in their succulent leaves and, thus, protect the metabolically active cellular compartments by restricting the excessive buildup of $\mathrm{Na}^{+}$ions in them [27]. Improved $\mathrm{K}^{+}$levels in leaves in ethanol-added salt-stressed soybean plants might assist in cell enlargement and optimal metabolic function, and preserve the structural integrity of proteins under ambient salt stress [40-42]. Moreover, increased $\mathrm{Mg}^{2+}$ levels in leaves may aid in protecting the chloroplast ultrastructure, translocating photoassimilates, and synthesizing chlorophylls, which has essential roles in the maintenance of optimum photosynthesis under saline conditions (Figure 6d) [43]. Thus, our results showed that the application of exogenous ethanol might efficiently ameliorate salinity-induced ion toxicity in soybean plants through the reduction in $\mathrm{Na}^{+}$accumulation and preferential nutrient allocation within different plant parts, which, in turn, promotes the overall growth performance of soybean plants.

\section{Materials and Methods}

\subsection{Plant species, Growth Environments, and Stress Treatments}

A high-yielding (1.8-2.1 tons $\mathrm{ha}^{-1}$ ) soybean (BARI Soybean-6) variety was chosen to assess the roles of ethanol in the mitigation of salt stress. Healthy seeds were surfacesterilized using sodium hypochlorite solution $(5 \%, v / v)$ containing Tween-20 solution $(0.2 \%, v / v)$ for $20 \mathrm{~min}$ followed by washing three times with distilled water $\left(\mathrm{dH}_{2} \mathrm{O}\right)$. Next, the sterilized seeds were immersed in $\mathrm{dH}_{2} \mathrm{O}$ at room temperature in the dark for $8 \mathrm{~h}$ for imbibition. The seeds were then covered with a wet cloth for $48 \mathrm{~h}$ to allow the radicle to emerge. The well-emerged radicles were then planted in a $2.5 \mathrm{~L}$ plastic pot $(17 \mathrm{~cm}$ height, $18 \mathrm{~cm}$ diameter) containing $2.5 \mathrm{~kg}$ of soil (eight radicles pot ${ }^{-1}$ ). Soils were prepared by mixing them with cow dung and sand in a weight-basis ratio of 2:1:0.5. Furadan, a wellknown pesticide, was added to the soil $\left(3.0 \mathrm{~g} \mathrm{~kg}^{-1}\right.$ of soil) to prevent soil-borne diseases. On the $10^{\text {th }}$ day after sowing, $200 \mathrm{~mL}$ of diluted urea $\left(4.0 \mathrm{~g} \mathrm{~L}^{-1}\right.$ of water $)$ was applied to each pot to ensure a sufficient supply of nitrogen fertilizer. Twelve-day-old seedlings at the vegetative V1 stage (completely formed first trifoliate) were divided into two groups (each group contained 3 pots). Both groups of pots were irrigated each day with tap water (control) and $8 \mathrm{dS} \mathrm{m}^{-1}$ (S1) and $16 \mathrm{dS} \mathrm{m}^{-1}$ (S2) of saline water $\left(200 \mathrm{~mL} \mathrm{pot}^{-1}\right.$ ) for seven days (7 times in total). During 12:15 p.m. to 13:00 p.m., one group of pots was simultaneously foliar-sprayed (20 mL to each pot) with $20 \mathrm{mM}$ of ethanol (Eth), while the pots from the remaining group were sprayed with tap water only $(20 \mathrm{~mL}$ to each pot). Therefore, the present study consisted of six treatments, including (i) water-sprayed control (Control), (ii) $20 \mathrm{mM}$ ethanol-sprayed control (Eth), (iii) water-sprayed $8 \mathrm{dS} \mathrm{m}^{-1}$ salt stress (S1), (iv) S1 + Eth, (v) water-sprayed $16 \mathrm{dS} \mathrm{m}^{-1}$ salt stress (S2), and (vi) S2 + Eth. Tween-20 $(0.2 \%, v / v)$ was used as a surfactant to ensure the maximal adherence of ethanol 
to the leaves. Nineteen-day-old seedlings were harvested to evaluate the performance of soybean under the above-mentioned conditions. The first trifoliate leaves were collected to determine various physiological and biochemical attributes. The experiment was repeated thrice to ensure that the results were accurate.

\subsection{Determination of Growth Parameters, RWC, and EL}

Following the method of Rahman et al.'s [27], three plants were randomly selected from each treatment to appraise the morphological features, including the height of shoot and DW of the shoots and roots. Roots from both control and salt-stressed plants were carefully detached from the soil, followed by washing according to the procedure described in Rahman et al. [6]. Afterward, primary root length was determined using a measuring scale. The EL of detached soybean leaves was determined following the method of Kim et al. [44]. Leaf RWC was assessed as described by Mostofa et al. [45] with a slight modification. The leaves of the first trifoliate were excised, and the fresh weight (FW) was immediately recorded. Excised leaves were hydrated to full turgid by immersing them in deionized $\mathrm{H}_{2} \mathrm{O}$ for $4 \mathrm{~h}$. The leaves were then gently pressed with tissue to remove adhered water, and the full turgid weight (TW) was taken. Oven-drying of the leaf samples was carried out at $80^{\circ} \mathrm{C}$ for $72 \mathrm{~h}$ followed by determination of DW. The percentage of RWC was calculated according to the equation: $\operatorname{RWC}(\%)=[(\mathrm{FW}-\mathrm{DW}) /(\mathrm{TW}-\mathrm{DW})] \times 100$.

\subsection{Measurement of Leaf Succulenceand Leaf Area per Trifoliate Leaf}

The leaf succulence of freshly harvested trifoliate leaves was measured using the protocols specified by Rahman et al. [6]. Total leaf area per trifoliate was determined using Carleton and Foote's [46] technique.

\subsection{Quantification of Ion Contents}

With the help of an atomic absorption spectrophotometer (PinAAcle 900H, Perkin Elmer, Waltham, MA, USA), the methods of Rahman et al. [14] was adopted to quantify the contents of $\mathrm{Na}^{+}, \mathrm{K}^{+}$, and $\mathrm{Mg}^{2+}$ in oven-dried samples of roots and leaves.

\subsection{Gas Exchange Parameters}

Between 10:30 a.m. and 12:30 p.m., the portable infrared gas analyzer system (LI6400XT, LI-COR Inc., Lincoln, NE, USA) was used to measure the $P_{n}, E, g_{s}$, and LT in the fully developed first trifoliate leaf (count from the plant's base). In addition, soybean leaves' WUEint and WUEins were determined using the formulae described by Rahman et al. [14].

\subsection{Determination of the Content of Photosynthetic Pigments and Pro}

The amounts of $\mathrm{Chl} a, \mathrm{Chl} b$, and total Chls, as well as the levels of carotenoids in freshly harvested soybean leaves, were spectrophotometrically estimated using the techniques described by Arnon [47], and Lichtenthaler and Wellbura [48], respectively. In addition, Pro content in freshly collected leaves was determined according to the procedure outlined by Bates et al. [49].

\subsection{Estimation of the Contents of Phenolics and Flavonoids}

The levels of total phenolics and total flavonoids in soybean leaf tissues were determined following the procedures reported by Ainsworth and Gillespie [50], and Zhishen et al. [51], respectively, with slight modifications. Briefly, soybean leaf samples (0.1 g) were homogenized in $1.5 \mathrm{~mL}$ of methanol $(100 \%)$ and centrifuged for $20 \mathrm{~min}$ at $4{ }^{\circ} \mathrm{C}$ at $11,500 \times g$. The collected supernatant was utilized following two different methodologies to quantify total phenolics and flavonoid contents. To determine total phenolics, $0.15 \mathrm{~mL}$ of $10 \%$ Folin-Ciocalteu's reagent was mixed with $0.3 \mathrm{~mL}$ of supernatant and kept at room temperature for $15 \mathrm{~min}$. After that, $0.6 \mathrm{~mL}$ of $700 \mathrm{mM} \mathrm{Na}{ }_{2} \mathrm{CO}_{3}$ was added to the above mixture and kept at room temperature for $120 \mathrm{~min}$. Finally, the absorbance was recorded at $765 \mathrm{~nm}$ using a UV-VIS spectrophotometer (GENESYS 10S, Thermo Scientific, San Jose, 
CA, USA). For the measurement of flavonoids, $0.15 \mathrm{~mL}$ of $5 \%$ sodium nitrite, $0.22 \mathrm{~mL}$ of $10 \% \mathrm{AlCl}_{3} \cdot 6 \mathrm{H}_{2} \mathrm{O}$, and $1.12 \mathrm{~mL}$ of $1.0 \mathrm{M} \mathrm{NaOH}$ were added to $0.3 \mathrm{~mL}$ of supernatant. The mixture was then shaken using a vortex mixer (XH-D Vortex Mixer, Shanghai Leewen Scientific Instrument Co. Ltd., Shanghai, China). Finally, the absorbance was measured at $510 \mathrm{~nm}$ using the above-mentioned spectrophotometer. Gallic acid and quercetin were used as standards for the determination of total phenolics and total flavonoids, respectively.

\subsection{Histochemical Detection of $\mathrm{O}_{2}{ }^{\bullet-}$ and $\mathrm{H}_{2} \mathrm{O}_{2}$, and Quantification of the Levels of $\mathrm{H}_{2} \mathrm{O}_{2}$ and $M D A$}

After 9 days of stress treatment, the histochemical detection of $\mathrm{O}_{2}{ }^{\bullet-}$ and $\mathrm{H}_{2} \mathrm{O}_{2}$ in soybean leaves was performed according to the methods of Mostofa and Fujita [52] with a slight modification. To stain $\mathrm{O}_{2}{ }^{\bullet-}$ and $\mathrm{H}_{2} \mathrm{O}_{2}$, fully grown first trifoliate soybean leaves were soaked in $0.05 \%(w / v)$ NBT and DAB solutions, respectively. The leaves immersed in NBT and DAB were incubated for $24 \mathrm{~h}$ under dark and light conditions, respectively. After incubation, the green color of the leaves was decolorized in boiling ethanol. The developed blue and brown spots indicated the presence of $\mathrm{O}_{2}{ }^{\bullet-}$ and $\mathrm{H}_{2} \mathrm{O}_{2}$, respectively. The photographs were taken by putting the stained soybean leaves on a whiteboard. The contents of $\mathrm{H}_{2} \mathrm{O}_{2}$ and lipid peroxidation product MDA in the first trifoliate leaves were quantified spectrophotometrically according to the methods reported by Yu et al. [53] and Kim et al. [44], respectively.

\subsection{Preparation of Enzyme Supernatants and Determination of Enzyme Activities}

The thorough procedures outlined by Rahman et al. [6] were followed for the preparation of enzyme extracts, and the estimation of the activities of CAT (EC 1.11.1.6), APX (EC: 1.11.1.11), POD (EC: 1.11.1.7), and GST (EC: 2.5.1.18).

\subsection{Quantification of Water-Soluble Proteins, Free Amino Acids, and Soluble Sugars}

The measurement of water-soluble protein contents in the enzyme extracts collected from the leaves of soybean plants were carried out following the spectrophotometric method of Bradford [54]. Total free amino acid and total soluble sugar contents were determined following the comprehensive procedures reported by Lee and Takahashi [55], and Somogyi [56], respectively.

\subsection{Statistical Analysis}

The obtained data were analyzed using one-way analysis of variance (ANOVA) with the employment of Statistix 10. The statistically significant differences $(p<0.05)$ among various treatments were shown by different letters following the least significant difference (LSD) test, using Statistix 10 software. Three biological replications $(n=3)$ were used to obtain the values (means \pm SEs) of each treatment, and they are presented in the Figure and Tables. Principle component analysis (PCA) was conducted using OriginPro 2021 software. A clustering heatmap was created with the normalized mean values of different parameters using R Studio 1.4.1717.

\section{Conclusions}

From our findings, we can conclude that ethanol can effectively ameliorate saltinduced growth retardation and biomass loss by modulating multiple physiological and biochemical processes. More specifically, ethanol application aided in (i) improving leaf succulence; (ii) attenuating uptake, transport, and accumulations of toxic $\mathrm{Na}^{+}$; (iii) shielding photosynthetic pigments degradation to improve photosynthetic performance; (iv) reducing oxidative stress and cellular damage by restricting the accumulations of excessive ROS; (v) augmenting CAT, APX, POD, and GST activities along with improving total phenolics and flavonoids levels; and (vi) enhancing compatible solutes accumulation in soybean under salt stress conditions. It is likely that application of exogenous ethanol might be a sustainable and cost-effective solution for reducing salinity-induced adverse effects on soy- 
bean production to support sustainable agriculture in saline-affected areas. Nevertheless, a further in-depth field investigation using a range of crop species, and different salinity regimes and modes of ethanol applications would be required to determine the advantageous role of ethanol in the proper management of salinity problems. As ethanol may quickly convert to other active metabolites, a comprehensive metabolite profiling might aid in determining the true contributor to ethanol-mediated salt tolerance in soybeans. Importantly, it would be interesting to assess if ethanol supplementation positively affects seed biochemical constituents and nutritional values in soybean, which might help us better address malnutrition issues in underdeveloped countries.

Author Contributions: Conceptualization, M.M.R. and M.G.M.; methodology, M.M.R. and A.K.D.; experimental work, A.K.D., T.R.A., M.M.R., M.R.I., S.S., P.K.G. and S.K.; software, T.R.A.; formal analysis, A.K.D. and T.R.A.; validation, M.M.R., M.G.M. and S.S.K.; investigation, M.M.R.; resources, M.A.R., T.A. and T.K.G.; data curation, A.K.D., T.R.A. and M.M.R.; writing-original draft preparation, M.M.R., A.K.D., T.R.A., S.S.K. and M.G.M.; writing-review and editing, M.G.M., L.S.-P.T. and M.M.R.; supervision, M.G.M. and M.M.R.; project administration, M.G.M.; funding acquisition, M.G.M. All authors have read and agreed to the published version of the manuscript.

Funding: This work was supported by the Ministry of Science and Technology (Project number: 146BS-2019), Bangladesh. The funders had no role in the study design, data collection and analysis, decision to publish, or preparation of the manuscript.

Data Availability Statement: The data presented in this study are available on request from the corresponding author. The data are not publicly available due to privacy.

Acknowledgments: The authors would like to thank the Department of Agronomy, BSMRAU for providing the portable photosynthetic machine (LI-6400XT).

Conflicts of Interest: The authors declare no conflict of interest.

\section{References}

1. Ferguson, J. Climate change and abiotic stress mechanisms in plants. Emerg. Top. Life Sci. 2019, 3, ETLS20180105.

2. Rahman, M.M.; Mostofa, M.G.; Keya, S.S.; Siddiqui, M.N.; Ansary, M.M.U.; Das, A.K.; Rahman, M.A.; Tran, L.S.-P. Adaptive mechanisms of halophytes and their potential in improving salinity tolerance in plants. Int. J. Mol. Sci. 2021, 22, 10733. [CrossRef]

3. Abiala, M.A.; Abdelrahman, M.; Burritt, D.J.; Tran, L.-S.P. Salt stress tolerance mechanisms and potential applications of legumes for sustainable reclamation of salt-degraded soils. Land Degrad. Dev. 2018, 29, 3812-3822. [CrossRef]

4. Zhang, J.L.; Shi, H. Physiological and molecular mechanisms of plant salt tolerance. Photosynth. Res. 2013, 115, 1-22. [CrossRef] [PubMed]

5. Qadir, M.; Quillérou, E.; Nangia, V.; Murtaza, G.; Singh, M.; Thomas, R.J.; Drechsel, P.; Noble, A.D. Economics of salt-induced land degradation and restoration. Nat. Resour. Forum 2014, 38, 282-295. [CrossRef]

6. Rahman, M.M.; Mostofa, M.G.; Rahman, M.A.; Islam, M.R.; Keya, S.S.; Das, A.K.; Miah, M.G.; Kawser, A.Q.M.R.; Ahsan, S.M.; Hashem, A.; et al. Acetic acid: A cost-effective agent for mitigation of seawater-induced salt toxicity in mung bean. Sci. Rep. 2019, 9, 15186. [CrossRef]

7. Zhao, C.; Zhang, H.; Song, C.; Zhu, J.-K.; Shabala, S. Mechanisms of plant responses and adaptation to soil salinity. Innov. 2020, 1, 100017. [CrossRef]

8. Arif, Y.; Singh, P.; Siddiqui, H.; Bajguz, A.; Hayat, S. Salinity induced physiological and biochemical changes in plants: An omic approach towards salt stress tolerance. Plant Physiol. Biochem. 2020, 156, 64-77. [CrossRef] [PubMed]

9. Mostofa, M.G.; Rahman, M.M.; Ansary, M.M.U.; Keya, S.S.; Abdelrahman, M.; Miah, M.G.; Tran, L.-S.P. Silicon in mitigation of abiotic stress-induced oxidative damage in plants. Crit. Rev. Biotechnol. 2021, 41, 918-934. [CrossRef]

10. Cao, D.; Li, Y.; Liu, B.; Kong, F.; Tran, L.-S.P. Adaptive mechanisms of soybean grown on salt-affected soils. Land Degrad. Dev. 2018, 29, 1054-1064. [CrossRef]

11. Sulieman, S.; Ha, C.; Esfahani, M.; Watanabe, Y.; Nishiyama, R.; Pham, C.; Nguyen, D.; Tran, L. DT2008: A promising new genetic resource for improved drought tolerance in soybean when solely dependent on symbiotic $\mathrm{N}_{2}$ fixation. In Crop Breeding: Bioinformatics and Preparing for Climate Change; Apple Academic Press: Williston, VT, USA, 2016; pp. $293-306$.

12. BBS. Yearbook of Agricultural Statistics of Bangladesh. In Planning Division; Government of the People's Republic of Bangladesh: Dhaka, Bangladesh, 2020.

13. DAE. Cultivation, Production and Yield Condition of Field Crop; Wing, F.S., Ed.; DAE: Khamarbari Dhaka, Bangladesh, 2021.

14. Rahman, M.; Mostofa, M.G.; Keya, S.S.; Rahman, A.; Das, A.K.; Islam, R.; Abdelrahman, M.; Bhuiyan, S.U.; Naznin, T.; Ansary, M.U.; et al. Acetic acid improves drought acclimation in soybean: An integrative response of photosynthesis, osmoregulation, mineral uptake and antioxidant defense. Physiol. Plant. 2021, 172, 334-350. [CrossRef] [PubMed] 
15. Farhad, I.S.M.; Rahman, M.A.; Jahan, E.; Azam, M.; Khan, N.R. Integrated nutrient management on soybean in a coastal charland of Bangladesh. Bangladesh Agron. J. 2017, 20, 77. [CrossRef]

16. Phang, T.-H.; Shao, G.; Lam, H.-M. Salt tolerance in soybean. J. Integr. Plant Biol. 2008, 50, 1196-1212. [CrossRef]

17. Chowdhury, M.M.U.; Sarker, M.J.U.; Choudhury, A.K.; Farhad, S.; Bhowal, S.; Hossain, K.M.F. Soybean Cultivation in Coastal Areas of Noakhali; On-Farm Research Division, Bangladesh Agricultural Research Institute: Gazipur, Bangladesh, 2013.

18. El Sabagh, A.; Hossain, A.; Barutçular, C.; Gormus, O.; Hussain, S.; Islam, M.S.; Alharaby, H.; Bamagoos, A.; Kumar, N.; Akdeniz, A.; et al. Effects of drought stress on the quality of major oilseed crops: Implications and possible mitigation strategies-A review. Appl. Ecol. Environ. Res. 2019, 17, 4019-4043. [CrossRef]

19. Kato-Noguchi, H. Low temperature acclimation mediated by ethanol production is essential for chilling tolerance in rice roots. Plant Signal. Behav. 2008, 3, 202-203. [CrossRef]

20. Nguyen, H.M.; Sako, K.; Matsui, A.; Suzuki, Y.; Mostofa, M.G.; Ha, C.V.; Tanaka, M.; Tran, L.-S.P.; Habu, Y.; Seki, M. Ethanol enhances high-salinity stress tolerance by detoxifying reactive oxygen species in Arabidopsis thaliana and rice. Front. Plant Sci. 2017, 8, 1001. [CrossRef]

21. Zörb, C.; Geilfus, C.-M.; Dietz, K.-J. Salinity and crop yield. Plant Biol. 2019, 21 (Suppl. 1), 31-38. [CrossRef]

22. Yasir, T.A.; Khan, A.; Skalicky, M.; Wasaya, A.; Rehmani, M.I.A.; Sarwar, N.; Mubeen, K.; Aziz, M.; Hassan, M.M.; Hassan, F.A.S.; et al. Exogenous sodium nitroprusside mitigates salt stress in lentil (Lens culinaris medik.) by affecting the growth, yield, and biochemical properties. Molecules 2021, 26, 2576. [CrossRef]

23. Rowe, R.N.; Farr, D.J.; Richards, B.A.J. Effects of foliar and root applications of methanol or ethanol on the growth of tomato plants (Lycopersicon esculentum Mill). N. Z. J. Crop Hortic. Sci. 1994, 22, 335-337. [CrossRef]

24. Yavarpanah, Z.; Alizadeh, M.; Seifi, E. Effects of foliar and root applications of hydro-alcoholic solutions on physiological and biochemical attributes and fruit yield and weight of strawberry. J. Plant Physiol. Breed. 2015, 5, 47-54.

25. Ashfaque, F.; Khan, M.I.R.; Khan, N.A. Exogenously applied $\mathrm{H}_{2} \mathrm{O}_{2}$ promotes proline accumulation, water relations, photosynthetic efficiency and growth of wheat (Triticum aestivum L.) under salt stress. Annu. Res. Rev. Biol. 2014, 4, 105-120. [CrossRef]

26. Mohsin, S.M.; Hasanuzzaman, M.; Bhuyan, M.H.M.B.; Parvin, K.; Fujita, M. Exogenous tebuconazole and trifloxystrobin regulates reactive oxygen species metabolism toward mitigating salt-induced damages in cucumber seedling. Plants 2019, 8, 428. [CrossRef]

27. Rahman, M.M.; Rahman, M.A.; Miah, M.G.; Saha, S.R.; Karim, M.A.; Mostofa, M.G. Mechanistic insight into salt tolerance of Acacia auriculiformis: The importance of ion selectivity, osmoprotection, tissue tolerance, and $\mathrm{Na}^{+}$exclusion. Front. Plant Sci. 2017, 8, 155. [CrossRef]

28. Soundararajan, P.; Manivannan, A.; Ko, C.H.; Muneer, S.; Jeong, B.R. Leaf physiological and proteomic analysis to elucidate silicon induced adaptive response under salt stress in Rosa hybrida 'rock fire'. Int. J. Mol. Sci. 2017, 18, 1768. [CrossRef] [PubMed]

29. Tekam, L.M.; Dany, P.M.; Victor, D.T.; Hartmut, S. Changes in plant growth, leaf relative water content and physiological traits in response to salt stress in peanut (Arachis hypogaea L.) varieties. Not. Bot. Horti Agrobot. Cluj-Napoca 2021, 49, 12049.

30. Considine, M.J.; Foyer, C.H. Oxygen and reactive oxygen species-dependent regulation of plant growth and development. Plant Physiol. 2021, 186, 79-92. [CrossRef]

31. Sharma, A.; Shahzad, B.; Rehman, A.; Bhardwaj, R.; Landi, M.; Zheng, B. Response of phenylpropanoid pathway and the role of polyphenols in plants under abiotic stress. Molecules 2019, 24, 2452. [CrossRef]

32. Šamec, D.; Karalija, E.; Šola, I.; Vujčić Bok, V.; Salopek-Sondi, B. The role of polyphenols in abiotic stress response: The influence of molecular structure. Plants 2021, 10, 118. [CrossRef] [PubMed]

33. Kiani, R.; Arzani, A.; Mirmohammady Maibody, S.A.M. Polyphenols, flavonoids, and antioxidant activity involved in salt tolerance in wheat, Aegilops cylindrica and their amphidiploids. Front. Plant Sci. 2021, 12, 493. [CrossRef] [PubMed]

34. Omari Alzahrani, F. Metabolic engineering of osmoprotectants to elucidate the mechanism(s) of salt stress tolerance in crop plants. Planta 2021, 253, 24. [CrossRef] [PubMed]

35. Abdel Latef, A.A.H.; Srivastava, A.K.; El-sadek, M.S.A.; Kordrostami, M.; Tran, L.-S.P. Titanium dioxide nanoparticles improve growth and enhance tolerance of broad bean plants under saline soil conditions. Land Degrad. Dev. 2018, 29, 1065-1073. [CrossRef]

36. Shafi, A.; Zahoor, I.; Mushtaq, U. Proline accumulation and oxidative stress: Diverse roles and mechanism of tolerance and adaptation under salinity stress. In Salt Stress, Microbes, and Plant Interactions: Mechanisms and Molecular Approaches; Springer: Berlin/Heidelberg, Germany, 2019; pp. 269-300.

37. El Moukhtari, A.; Cabassa-Hourton, C.; Farissi, M.; Savouré, A. How does proline treatment promote salt stress tolerance during crop plant development? Front. Plant Sci. 2020, 11, 1127. [CrossRef]

38. Batista-Silva, W.; Heinemann, B.; Rugen, N.; Nunes-Nesi, A.; Araújo, W.L.; Braun, H.P.; Hildebrandt, T.M. The role of amino acid metabolism during abiotic stress release. Plant Cell Environ. 2019, 42, 1630-1644. [CrossRef] [PubMed]

39. Khan, N.; Ali, S.; Zandi, P. Role of sugars, amino acids and organic acids in improving plant abiotic stress tolerance. Pak. J. Bot. 2020, 52, 355-363. [CrossRef]

40. Benito, B.; Haro, R.; Amtmann, A.; Cuin, T.A.; Dreyer, I. The twins $\mathrm{K}^{+}$and $\mathrm{Na}^{+}$in plants. J. Plant Physiol. 2014, $171,723-731$. [CrossRef]

41. Parida, A.K.; Veerabathini, S.K.; Kumari, A.; Agarwal, P.K. Physiological, anatomical and metabolic implications of salt tolerance in the halophyte Salvadora persica under hydroponic culture condition. Front. Plant Sci. 2016, 7, 351. [CrossRef]

42. Assaha, D.V.M.; Ueda, A.; Saneoka, H.; Al-Yahyai, R.; Yaish, M.W. The role of $\mathrm{Na}^{+}$and $\mathrm{K}^{+}$transporters in salt stress adaptation in glycophytes. Front. Physiol. 2017, 8, 509. [CrossRef] 
43. Tränkner, M.; Tavakol, E.; Jákli, B. Functioning of potassium and magnesium in photosynthesis, photosynthate translocation and photoprotection. Physiol. Plant 2018, 163, 414-431. [CrossRef]

44. Kim, T.Y.; Ku, H.; Lee, S.-Y. Crop enhancement of cucumber plants under heat stress by shungite carbon. Int. J. Mol. Sci. 2020, 21, 4858. [CrossRef]

45. Mostofa, M.G.; Hossain, M.A.; Fujita, M. Trehalose pretreatment induces salt tolerance in rice (Oryza sativa L.) seedlings: Oxidative damage and co-induction of antioxidant defense and glyoxalase systems. Protoplasma 2015, 252, 461-475. [CrossRef]

46. Carleton, A.E.; Foote, W.H. A Comparison of methods for estimating total leaf area of barley plants. Crop Sci. 1965, 5, 602-603. [CrossRef]

47. Arnon, D.I. Copper enzymes in isolated chloroplasts. polyphenoloxidase in Beta vulgaris. Plant Physiol. 1949, 24, 1-15. [CrossRef]

48. Lichtenthaler, H.K.; Wellburn, A.R. Determinations of total carotenoids and chlorophylls $a$ and $b$ of leaf extracts in different solvents. Biochem. Soc. Trans. 1983, 11, 591-592. [CrossRef]

49. Bates, L.S.; Waldren, R.P.; Teare, I.D. Rapid determination of free proline for water-stress studies. Plant Soil 1973, 39, $205-207$. [CrossRef]

50. Ainsworth, E.A.; Gillespie, K.M. Estimation of total phenolic content and other oxidation substrates in plant tissues using Folin-Ciocalteu reagent. Nat. Protoc. 2007, 2, 875-877. [CrossRef] [PubMed]

51. Zhishen, J.; Mengcheng, T.; Jianming, W. The determination of flavonoid contents in mulberry and their scavenging effects on superoxide radicals. Food Chem. 1999, 64, 555-559. [CrossRef]

52. Mostofa, M.G.; Fujita, M. Salicylic acid alleviates copper toxicity in rice (Oryza sativa L.) seedlings by up-regulating antioxidative and glyoxalase systems. Ecotoxicology 2013, 22, 959-973. [CrossRef]

53. Yu, C.-W.; Murphy, T.; Lin, C.-H. Hydrogen peroxide-induced chilling tolerance in mung beans mediated through ABAindependent glutathione accumulation. Funct. Plant Biol. 2003, 30, 955-963. [CrossRef] [PubMed]

54. Bradford, M.M. A rapid and sensitive method for the quantitation of microgram quantities of protein utilizing the principle of protein-dye binding. Anal. Biochem. 1976, 72, 248-254. [CrossRef]

55. Lee, Y.P.; Takahashi, T. An improved colorimetric determination of amino acids with the use of ninhydrin. Anal. Biochem. 1966, 14, 71-77. [CrossRef]

56. Somogyi, M. Notes on sugar determination. J. Biol. Chem. 1952, 195, 19-23. [CrossRef] 